\title{
Adaptation of generalized Hill inequalities to anisotropic elastic symmetries
}

\author{
Ç. Dinçkal ${ }^{1}$ * \\ ${ }^{1 *}$ Department of Civil Engineering, Çankaya University, TURKEY \\ "Corresponding Author: e-mail: cigdemdinckal2004@yahoo.com,, Tel +90-505-6709413,
}

\begin{abstract}
Mechanical and elastic behaviors of anisotropic materials are investigated in an innovative way. This is based on generalized Hill inequalities. From different type of anisotropic elastic symmetries, numerical examples are given. Constructing bounds on effective eigenvalues provides a deeper understanding about mechanical behavior of anisotropic materials. Generalized Hill inequalities are adapted to all anisotropic elastic symmetries. The materials selected from the same symmetry type which have larger interval between the bounds, are more anisotropic whereas smaller interval between the bounds, are closer to isotropy. Besides it is proved that there are relations between bulk and shear modulus and eigenvalues of cubic and isotropic symmetry and by these relations, two linear invariants are found out.
\end{abstract}

Keywords: Generalized Hill Inequalities, Elastic Constants, Anisotropic Elastic Symmetries, Anisotropy, Bounds..

\section{Introduction}

A material is said to be isotropic if its mechanical and elastic properties are the same in all directions. When this is not true, the material is said to be anisotropic. Anisotropic materials become the material of choice in a variety of engineering applications in the last century. Many materials are anisotropic and inhomogeneous due to the varying composition of their constituents. For instance, polycrystalline materials generally show an elastic anisotropy due to texture and the anisotropy of single crystallites. The polycrystalline and composite materials which show high anisotropy are used in many applications in industry. According to Hill (1963); the material with triclinic or greater symmetry can be either a polycrystalline material or a composite material or a single crystalline material. In polycrystalline materials, the relation between the elastic properties of single crystals and of 'quasiisotropic' bodies made up of a large number of small single crystallites disposed at all possible orientations has been investigated by Reuss and Voigt. Voigt took the averages of stiffness tensor $\left(c_{i j}\right)$ of the crystallites while Reuss took the averages of compliance tensor $\left(s_{i j}\right)$ of the crystallites. The Voigt bound is based on an assumed uniform strain, in other words, Voigt assumes the same deformation in all the grains (uniform deformation) and the Reuss bound is based on an assumed uniform stress which means the same stress in all the grains by Mehrabadi and Cowin (1995).

In literature, Dinçkal and Akgöz (2010) decomposed elastic constant tensor into orthogonal parts in order to investigate the mechanical and elastic behavior of an anisotropic material and determine the anisotropy degree of that material. These processes are done by various decomposition methods. But this work presents a different method by developing generalized Hill inequalities and adapting them to anisotropic materials from different symmetry types. In this paper, mechanical and elastic behaviour of anisotropic materials are investigated based on generalized Hill inequalities. These are adapted to all anisotropic elastic symmetries. Kelvin formulation of the anisotropic Hook's law is described. The bounds are constructed numerically in order to determine the sets of effective eigenvalues for all material symmetry types. Lastly, we outline significant effects on many applications in different fields.

\section{A Tensorial Presentation of the Kelvin Formulation}

The anisotropic form of Hooke's law in linear elasticity is often written in indicial notation as 
$T_{i j=} c_{i j k m} E_{k m}$

where $T_{i j}$ are components of stress tensor, $E_{k m}$ are components of infinitesimal strain tensor and $C_{i j k m}$ are the components of elasticity tensor. In other words, $C_{i j k m}$ are the components of a fourth-rank tensor called the elastic constant tensor (stiffness tensor) and $i, j, k, m=1,2,3$. It is written as a linear transformation in six dimensions, Hooke's law has the representation $\mathbf{T}=\mathbf{c E}$ and in Voigt notation, it is represented as follows

$\left[\begin{array}{l}T_{11} \\ T_{22} \\ T_{33} \\ T_{23} \\ T_{13} \\ T_{12}\end{array}\right]=\left[\begin{array}{llllll}c_{11} & c_{12} & c_{13} & c_{14} & c_{15} & c_{16} \\ c_{12} & c_{22} & c_{23} & c_{24} & c_{25} & c_{26} \\ c_{13} & c_{23} & c_{33} & c_{34} & c_{35} & c_{36} \\ c_{14} & c_{24} & c_{34} & c_{44} & c_{45} & c_{46} \\ c_{15} & c_{25} & c_{35} & c_{45} & c_{55} & c_{56} \\ c_{16} & c_{26} & c_{36} & c_{46} & c_{56} & c_{66}\end{array}\right]\left[\begin{array}{c}E_{11} \\ E_{22} \\ E_{33} \\ 2 E_{23} \\ 2 E_{13} \\ 2 E_{12}\end{array}\right]$

The relationships of the components of $C_{i j k m}$ to the components of the symmetric matrix $\mathbf{C}$ are given in Table 2.1. By introducing new notation, equation (2.2) can be rewritten in the form $\hat{T}=\hat{c} \hat{E}$, where the shearing components of these new six dimensional stress and strain vectors which are denoted by $\hat{T}$ and $\hat{E}$, respectively. They are multiplied by $\sqrt{2}, \hat{C}$ a new six- by-six matrix is obtained in work of Mehrabadi and Cowin (1995). The matrix form of $\hat{T}=\hat{c} \hat{E} \quad$ which is in terms Voigt in double index notation is represented as follows:

$\left[\begin{array}{c}T_{11} \\ T_{22} \\ T_{33} \\ \sqrt{2} T_{23} \\ \sqrt{2} T_{13} \\ \sqrt{2} T_{12}\end{array}\right]=\left[\begin{array}{cccccc}c_{11} & c_{12} & c_{13} & \sqrt{2} c_{14} & \sqrt{2} c_{15} & \sqrt{2} c_{16} \\ c_{12} & c_{22} & c_{23} & \sqrt{2} c_{24} & \sqrt{2} c_{25} & \sqrt{2} c_{26} \\ c_{13} & c_{23} & c_{33} & \sqrt{2} c_{34} & \sqrt{2} c_{35} & \sqrt{2} c_{36} \\ \sqrt{2} c_{14} & \sqrt{2} c_{15} & \sqrt{2} c_{16} & 2 c_{44} & 2 c_{45} & 2 c_{46} \\ \sqrt{2} c_{24} & \sqrt{2} c_{25} & \sqrt{2} c_{26} & 2 c_{45} & 2 c_{55} & 2 c_{56} \\ \sqrt{2} c_{34} & \sqrt{2} c_{35} & \sqrt{2} c_{36} & 2 c_{46} & 2 c_{56} & 2 c_{66}\end{array}\right]\left[\begin{array}{c}E_{11} \\ E_{22} \\ E_{33} \\ \sqrt{2} E_{23} \\ \sqrt{2} E_{23} \\ \sqrt{2} E_{23}\end{array}\right]$

The matrix $\hat{C}$ is called the matrix of elastic constants and its inverse $\hat{s}, \quad \hat{E}=\hat{s} \hat{T} \quad \hat{s}=\hat{C}^{-1}$ is called the compliance matrix. A table relating these various notations for the specific elastic constants is given in Table 2.1. The symmetric matrices $\hat{c}$ and $\hat{S}$ can be shown to represent the components of a second-rank tensor in a six dimensional space. In this thesis, these matrices are used in the following chapters. Since the components of the matrix $\mathbf{C}$ appearing in equation (2.2) do not form a tensor (Mehrabadi and Cowin (1995)).

Table 2.1: The elasticity and compliance in different notations

\begin{tabular}{|l|l|l|l|l|l|}
\hline 1 & 2 & 3 & 1 & 2 & 3 \\
\hline$C_{1111}$ & $C_{11}$ & $\hat{c}_{11}$ & $S_{1111}$ & $S_{11}$ & $\hat{s}_{11}$ \\
\hline$C_{2222}$ & $C_{22}$ & $\hat{c}_{22}$ & $S_{2222}$ & $S_{22}$ & $\hat{s}_{22}$ \\
\hline$C_{3333}$ & $C_{33}$ & $\hat{c}_{33}$ & $S_{3333}$ & $S_{33}$ & $\hat{s}_{33}$ \\
\hline
\end{tabular}




\begin{tabular}{|c|c|c|c|c|c|}
\hline$C_{1122}$ & $C_{12}$ & $\hat{c}_{12}$ & $S_{1122}$ & $S_{12}$ & $\hat{s}_{12}$ \\
\hline$C_{1133}$ & $C_{13}$ & $\hat{C}_{13}$ & $S_{1133}$ & $S_{13}$ & $\hat{s}_{13}$ \\
\hline$C_{2233}$ & $c_{23}$ & $\hat{C}_{23}$ & $S_{2233}$ & $S_{23}$ & $\hat{s}_{23}$ \\
\hline$c_{2323}$ & $C_{44}$ & $\frac{1}{2} \hat{C}_{44}$ & $S_{2323}$ & $\frac{1}{4} S_{44}$ & $\frac{1}{2} \hat{S}_{44}$ \\
\hline$C_{1313}$ & $C_{55}$ & $\frac{1}{2} \hat{c}_{55}$ & $S_{1313}$ & $\frac{1}{4} S_{55}$ & $\frac{1}{2} \hat{S}_{55}$ \\
\hline$C_{1212}$ & $c_{66}$ & $\frac{1}{2} \hat{C}_{66}$ & $S_{1212}$ & $\frac{1}{4} S_{66}$ & $\frac{1}{2} \hat{S}_{66}$ \\
\hline$c_{1323}$ & $C_{54}$ & $\frac{1}{2} \hat{c}_{54}$ & $S_{1323}$ & $\frac{1}{4} S_{54}$ & $\frac{1}{2} \hat{S}_{54}$ \\
\hline$C_{1312}$ & $C_{56}$ & $\frac{1}{2} \hat{C}_{56}$ & $S_{1312}$ & $\frac{1}{4} S_{56}$ & $\frac{1}{2} \hat{S}_{56}$ \\
\hline$C_{1223}$ & $C_{64}$ & $\frac{1}{2} \hat{C}_{64}$ & $S_{1223}$ & $\frac{1}{4} S_{64}$ & $\frac{1}{2} \hat{S}_{64}$ \\
\hline$C_{2311}$ & $C_{41}$ & $\frac{1}{\sqrt{2}} \hat{C}_{41}$ & $S_{2311}$ & $\frac{1}{2} S_{41}$ & $\frac{1}{\sqrt{2}} \hat{s}_{411}$ \\
\hline$C_{1311}$ & $C_{51}$ & $\frac{1}{\sqrt{2}} \hat{c}_{51}$ & $S_{1311}$ & $\frac{1}{2} S_{51}$ & $\frac{1}{\sqrt{2}} \hat{s}_{51}$ \\
\hline$C_{1211}$ & $C_{61}$ & $\frac{1}{\sqrt{2}} \hat{c}_{61}$ & $S_{1211}$ & $\frac{1}{2} S_{61}$ & $\frac{1}{\sqrt{2}} \hat{S}_{61}$ \\
\hline$c_{2322}$ & $C_{42}$ & $\frac{1}{\sqrt{2}} \hat{c}_{42}$ & $S_{2322}$ & $\frac{1}{2} S_{42}$ & $\frac{1}{\sqrt{2}} \hat{s}_{42}$ \\
\hline$C_{1322}$ & $C_{52}$ & $\frac{1}{\sqrt{2}} \hat{c}_{52}$ & $S_{1322}$ & $\frac{1}{2} S_{52}$ & $\frac{1}{\sqrt{2}} \hat{S}_{52}$ \\
\hline$C_{1222}$ & $c_{62}$ & $\frac{1}{\sqrt{2}} \hat{c}_{62}$ & $S_{1222}$ & $\frac{1}{2} S_{62}$ & $\frac{1}{\sqrt{2}} \hat{S}_{62}$ \\
\hline$C_{2333}$ & $C_{43}$ & $\frac{1}{\sqrt{2}} \hat{C}_{43}$ & $S_{2333}$ & $\frac{1}{2} S_{43}$ & $\frac{1}{\sqrt{2}} \hat{S}_{43}$ \\
\hline$C_{1333}$ & $C_{53}$ & $\frac{1}{\sqrt{2}} \hat{c}_{53}$ & $S_{1333}$ & $\frac{1}{2} S_{53}$ & $\frac{1}{\sqrt{2}} \hat{s}_{53}$ \\
\hline$c_{1233}$ & $c_{63}$ & $\frac{1}{\sqrt{2}} \hat{c}_{63}$ & $S_{1233}$ & $\frac{1}{2} S_{63}$ & $\frac{1}{\sqrt{2}} \hat{S}_{63}$ \\
\hline
\end{tabular}

In Table 2.1, column 1 illustrates the Voigt notation of these quantities as fourth rank tensor components in a three dimensional Cartesian space. Column 2 represents the same Voigt matrix in double index notation. Column 3 illustrates the Kelvin-inspired notation for these quantities as second rank tensor components in a six dimensional Cartesian space.

The eigenvalues of the matrix $\hat{c}(\hat{s})$ are the six numbers $\lambda\left(\frac{1}{\lambda}\right)$ satisfying the following equations:

$(\hat{c}-\lambda I) \hat{N}=0$

$\left(\hat{s}-\left(\frac{1}{\lambda}\right) I\right) \hat{N}=0$

Where $\mathbf{I}$ is the identity matrix and same size with matrices $\hat{C}$ and $\hat{S}$. The vectors $\hat{N}$ represent the normalized eigenvectors of $\hat{c}$ (or $\hat{s}$ ). The normalized $\hat{N}$ are expressed in terms of the six dimensional strain and stress vectors by

$$
\hat{E}=\hat{N}|\hat{E}|, \quad \hat{T}=\hat{N}|\hat{T}|, \quad \quad|\hat{E}|^{2}=\hat{E} \bullet \hat{E}, \quad|\hat{T}|^{2}=\hat{T} \bullet \hat{T}, \quad \hat{N} \bullet \hat{N}=1 .
$$


Since $\hat{C}$ (or $\hat{S}$ ) is positive definite (Ting (1996)), it has six positive eigenvalues. These eigenvalues are called the Kelvin moduli and are denoted by $\lambda_{i}, \mathrm{i}=1,2,3,4,5,6$. They are ordered (if possible) by the inequalities $\lambda_{1} \geq \ldots . \lambda_{6}>0$. The eigenvalues of $\hat{\mathrm{s}}$ are the inverses of the eigenvalues of $\hat{c}$. There exists six eigentensors of stress, denoted by $\hat{T}^{(k)}, k=1,2,3,4,5,6$ in the six dimensional space, or by $T^{(k)}$ in the three dimensional space and six eigentensors of strain, denoted by $\hat{E}^{(k)}$ and $E^{(k)}, k=1,2,3,4,5,6$, respectively, which are related by the following six equations,

$\hat{T}^{(k)}=\lambda_{k} \hat{E}^{(k)}, \quad T^{(k)}=\lambda_{k} E^{(k)}, \quad k=1,2,3,4,5,6$.

The strain energy $\sum$,

$$
\sum=\frac{1}{2} \hat{E} \bullet \hat{c} \hat{E}=\frac{1}{2} \hat{T} \bullet \hat{c} \hat{T}
$$

A result based on the stress-strain relation, $\hat{T}=\hat{c} \hat{E}$ is easily converted to a result based on the strain-stress relation, $\hat{E}=\hat{s} \hat{T}$ It is done simply by interchanging $\hat{T}$ and $\hat{E}, \hat{C}$ and $\hat{S}$, respectively. So, while the results presented in this thesis optimize with respect to strain states, the results for optimization with respect to stress states are obtained by interchanging the following terms; $\hat{T}$ and $\hat{E}$, $\hat{c}$ and $\hat{s}$, and $\lambda_{i}$ and $\left(1 / \lambda_{i}\right)$.

Such simplicity of notation is possible with Kelvin inspired notation.

\section{Generalized Hill Inequalities}

Hill (1963) notes that the average strain energy in any region can be calculated from the average stress and the average strain. So the average strain denoted by $\hat{E}^{A}$, and the average stress denoted by $\hat{T}^{A}$ the average strain energy has the dual representation $2 \sum^{A}=\hat{E}^{A} \bullet \hat{c}^{e f f} \hat{E}^{A}=\hat{T}^{A} \bullet \hat{s}^{e f f} \hat{T}^{A}$

where the definitions of $\hat{C}^{\text {eff }}$ and $\hat{S}^{\text {eff }}$ have been employed,

$$
\hat{T}^{A}=\hat{c}^{e f f} \hat{E}^{A}, \quad \hat{E}^{A}=\hat{s}^{e f f} \hat{T}^{A},
$$

According to the familiar principle of minimum potential energy for an elastic continuum, the actual strain energy in the mixture does not exceed the energy of any unequilibrated state of distortion with the same surface displacements. When these are compatible with average strain $\hat{E}^{A}$, it can be taken as a comparison fictitious state which has an energy density $\hat{E}^{A} \cdot \hat{C}^{V} \hat{E}^{A}$. So by considering a representative volume under such surface constraints and by using equation (3.1), we get

$$
E^{A} \bullet \hat{c}^{e f f} \hat{E}^{A} \leq \hat{E}^{A} \bullet \hat{c}^{V} \hat{E}^{A}
$$

Similarly, by considering loading under surface tractions compatible with average stress, and applying the dual principle of minimum potential energy, we get

$\hat{T}^{A} \bullet \hat{s}^{e f f} \hat{T}^{A} \leq \hat{T}^{A} \bullet \hat{s}^{R} \hat{T}^{A}$

By using the inequalities (3.3) and (3.4), generalized Hill inequalities can be formed. The argument is simple and it depends upon the following theorem; 
$N \times N$ Matrices $A$ and $B$ are taken. These matrices satisfy the inequality of quadratic forms, $x \cdot A x \leq x \cdot B x$, for all vectors $x$ in the $N$-dimensional space, then the eigenvalues of $A$ and $B, \lambda_{i}^{A}$ and $\lambda_{i}^{B}, i=1, \ldots, N$ respectively, satisfy the inequalities $\lambda_{i}^{A} \leq \lambda_{i}^{B}, i=1, \ldots, N$.

$A$ and $B$ are real and positive definite matrices. So both of them must satisfy the following conditions (Strang (1976)):

1. $x^{T} A x>0$ and $x^{T} B x>0$. In indicial notation, $x_{i}^{T} A x_{i}>0$ and $x_{i}^{T} B x_{i}>0$ for all nonzero vectors $\mathrm{x}$ (where superscript $T$ indicates transpose).

2. The eigenvalues of $\mathrm{A}, \lambda^{A}>0$ and $\lambda^{B}>0$.

3. In indicial notation $x_{i}^{T} x_{i}=1$ (it is also satisfied for orthogonality condition).

The eigenvalues of the matrices A and B should satisfy the following equations:

$A x=\lambda^{A} x$ and $B x=\lambda^{B} x$,

where $\lambda^{A}$ denotes the eigenvalues of $\mathrm{A}, \quad \lambda^{B}$ denotes eigenvalues of $\mathrm{B}$ and $\mathrm{x}$ represents all eigenvectors in the $N$ dimensional space. The indicial form of equation (3.5) is

$$
A x_{i}=\lambda_{i}^{A} x_{i} \text { and } B x_{i}=\lambda_{i}^{B} x_{i}
$$

In order to prove this theorem, the following operations are performed. If we multiply all terms by $x_{i}^{T}$ in equation (3.6) then we obtain

$$
x_{i}^{T} A x_{i}=x_{i}^{T} \lambda_{i}^{A} x_{i} \quad \text { and } \quad x_{i}^{T} B x_{i}=x_{i}^{T} \lambda_{i}^{B} x_{i} .
$$

Since the multiplication has commutative property, equation (3.7) takes the form

$$
x_{i}^{T} \lambda_{i}^{A} x_{i}=x_{i}^{T} x_{i} \lambda_{i}^{A} \quad \text { and } \quad x_{i}^{T} \lambda_{i}^{B} x_{i}=x_{i}^{T} x_{i} \lambda_{i}^{B} .
$$

Since, $x_{i}^{T} x_{i}=1$, then equation (3.8) becomes

$$
x_{i}^{T} \lambda_{i}^{A} x_{i}=\lambda_{i}^{A} \quad \text { and } \quad x_{i}^{T} \lambda_{i}^{B} x_{i}=\lambda_{i}^{B} .
$$

By using equation (3.9), we obtain the inequality

$$
\lambda_{i}^{A} \leq \lambda_{i}^{B} .
$$

The result of inequalities (3.3) and (3.4) must hold for each eigenvalue, so from the theorem above, the following inequalities can be obtained

$$
\lambda_{i}^{\text {eff }} \leq \lambda_{i}^{V} \quad, \quad \lambda_{i}^{R} \leq \lambda_{i}^{\text {eff }}\left(\frac{1}{\lambda_{i}^{\text {eff }}} \leq \frac{1}{\lambda_{i}^{R}}\right) . \quad \text { for } i=1,2,3,4,5,6 .
$$

The inequalities (3.11) may be combined in the final result

$$
\lambda_{i}^{R} \leq \lambda_{i}^{\text {eff }} \leq \lambda_{i}^{V}, \quad \text { for } \quad i=1,2,3,4,5,6 \text {. }
$$


The above inequalities are referred as the generalized Hill inequalities. These inequalities will be used later in section 5 and section 6 in order to construct the bounds for all anisotropic symmetry types which are isotropic, cubic, transversely isotropic, tetragonal, trigonal, orthotropic, monoclinic and triclinic.

\section{Adaptation of Generalized Hill Inequalities to Various Symmetries}

Generalized Hill inequalities can be adapted to any material which shows an anisotropic elastic symmetry. In order to derive the inequalities, eigenvalues of each symmetry type should be calculated. These eigenvalues are Voigt eigenvalues and Reuss eigenvalues which are denoted by $\lambda^{V}$ and $\lambda^{R}$ respectively. Voigt eigenvalues are the upper bounds while Reuss eigenvalues are the lower bounds for all symmetries. $\hat{C}^{V}$ (Voigt elastic constant tensor) matrices which represent the Voigt matrices, are used to calculate the Voigt eigenvalues. $\hat{C}^{V}$ are formed by averaging the $\hat{C}^{\text {Tric }}$ (elastic constant tensor) elements of triclinic

symmetry. In similar fashion, $\hat{S}^{R}$ (Reuss elastic compliances) matrices which represent the Reuss matrices, are used to calculate the Reuss eigenvalues.

\subsection{For Cubic Media}

In cubic symmetry, four three-fold axes arranged like the body diagonals of a cube. Cubic symmetry has three distinct eigenvalues. They are of multiplicity one, two, three. For this symmetry type, equation (2.4) takes the following forms

$\left(\hat{C}^{V, C u b}-\lambda I\right) \hat{N}=0$

$\left[\begin{array}{cccccc}\hat{c}_{11}^{V, \text { Cub }}-\lambda & \hat{c}_{12}^{V, \text { Cub }} & \hat{c}_{12}^{V, \text { Cub }} & 0 & 0 & 0 \\ \hat{c}_{12}^{V, \text { Cub }} & \hat{c}_{11}^{V, \text { Cub }}-\lambda & \hat{c}_{12}^{V, \text { Cub }} & 0 & 0 & 0 \\ \hat{c}_{12}^{V, \text { Cub }} & \hat{c}_{12}^{V, \text { Cub }} & \hat{c}_{11}^{V, \text { Cub }}-\lambda & 0 & 0 & 0 \\ 0 & 0 & 0 & \hat{c}_{44}^{V, C u b}-\lambda & 0 & 0 \\ 0 & 0 & 0 & 0 & \hat{c}_{44}^{V, C u b}-\lambda & 0 \\ 0 & 0 & 0 & 0 & 0 & \hat{c}_{44}^{V, C u b}-\lambda\end{array}\right]\left[\begin{array}{c}\hat{N}_{1} \\ \hat{N}_{2} \\ \hat{N}_{3} \\ \hat{N}_{4} \\ \hat{N}_{5} \\ \hat{N}_{6}\end{array}\right]=0$

The elements ( $\hat{C}^{V, C u b}$ ) of matrix can be expressed in terms of the elements ( $\hat{C}^{\text {Tric }}$, elastic stiffnesses) of the triclinic symmetry by using the following relations:

$\hat{c}_{11}^{V, C u b}=\frac{1}{3}\left(\hat{c}_{11}^{\text {Tric }}+\hat{c}_{22}^{\text {Tric }}+\hat{c}_{33}^{\text {Tric }}\right), \hat{c}_{12}^{V, \text { Cub }}=\frac{1}{3}\left(\hat{c}_{12}^{\text {Tric }}+\hat{c}_{23}^{\text {Tric }}+\hat{c}_{13}^{\text {Tric }}\right), \hat{c}_{44}^{V, \text { Cub }}=\frac{1}{3}\left(\hat{c}_{44}^{\text {Tric }}+\hat{c}_{55}^{\text {Tric }}+\hat{c}_{66}^{\text {Tric }}\right)$.

Then Voigt eigenvalues for cubic symmetry are

$\lambda_{1}^{V, C u b}=\left(\hat{c}_{11}^{V, C u b}\right)+2\left(\hat{c}_{12}^{V, C u b}\right)=3 K^{V, C u b}$,

where $K^{V, C u b}=\frac{\hat{c}_{11}^{V, C u b}+2 \hat{c}_{12}^{V, C u b}}{3}$ (bulk modulus of Voigt for cubic symmetry). First invariant ( $I_{1}$ ) is found as $9 K^{V, C u b}$.

This is trace of elastic constant matrix for cubic symmetry. Second and third eigenvalues represented by $\lambda_{(2,3)}^{V, \text { Cub }}$. and forth, fifth and sixth eigenvalues are represented by $\lambda_{(4,5,6)}^{V, C u b}$. are defined as follows

$\lambda_{(2,3)}^{V, \text { Cub }}=\hat{c}_{11}^{V, \text { Cub }}-\hat{c}_{12}^{V, \text { Cub }}, \lambda_{(4,5,6)}^{V, \text { Cub }}=2 \hat{c}_{44}^{V, \text { Cub }}$. 
For this symmetry type, equation (2.5) takes the following forms

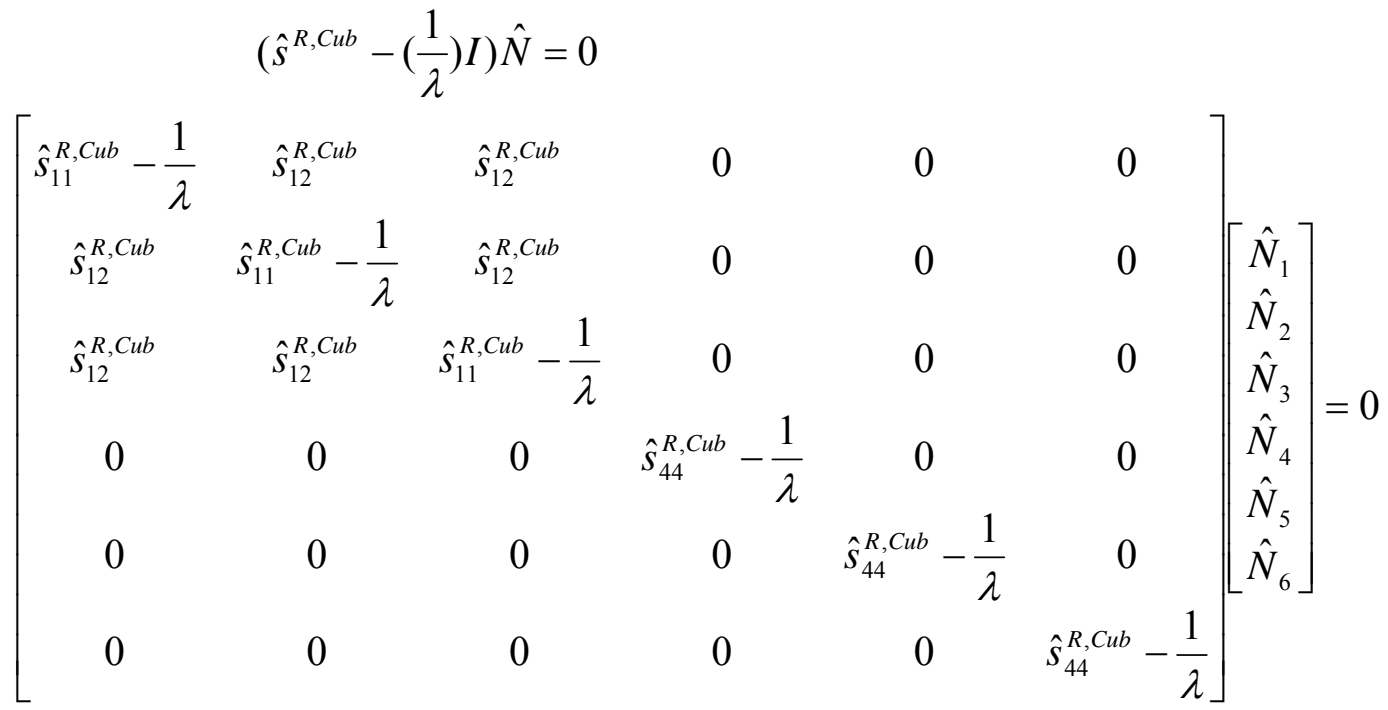

The elements ( $\hat{S}^{R, C u b}$ ) of matrix can be expressed in terms of the elements ( $\hat{S}^{\text {Tric }}$, elastic compliances) of the triclinic symmetry by using the following terms:

$\hat{S}_{11}^{R, \text { Cub }}=\frac{\left(\hat{S}_{11}^{\text {Tric }}+\hat{S}_{22}^{\text {Tric }}+\hat{S}_{33}^{\text {Tric }}\right)}{3}, \hat{S}_{12}^{R, \text { Cub }}=\frac{\left(\hat{S}_{12}^{\text {Tric }}+\hat{S}_{23}^{\text {Tric }}+\hat{S}_{31}^{\text {Tric }}\right)}{3}, \hat{s}_{44}^{R, \text { Cub }}=\frac{\left(\hat{S}_{44}^{\text {Tric }}+\hat{S}_{55}^{\text {Tric }}+\hat{S}_{66}^{\text {Tric }}\right)}{3}$.

The Reuss eigenvalues for cubic symmetry are

$\frac{1}{\lambda_{1}^{R, C u b}}=\hat{s}_{11}^{R, C u b}+2 \hat{s}_{12}^{R, C u b}, \frac{1}{\lambda_{(2,3)}^{R, C u b}}=\left(\hat{s}_{11}^{R, C u b}-\hat{s}_{12}^{R, C u b}\right), \frac{2}{\lambda_{(4,5,6)}^{R, C u b}}=\hat{s}_{44}^{V, C u b}$.

The generalized Hill inequalities for cubic symmetry are

$\lambda_{1}^{R, C u b} \leq \lambda_{1}^{e f f, C u b} \leq \lambda_{1}^{V, C u b}, \quad \lambda_{(2,3)}^{R, C u b} \leq \lambda_{(2,3)}^{e f f, C u b} \leq \lambda_{(2,3)}^{V, C u b}, \quad \lambda_{(4,5,6)}^{R, C u b} \leq \lambda_{(4,5,6)}^{\text {eff,Cub }} \leq \lambda_{(4,5,6)}^{V, \text {. }}$.

\subsection{For Isotropic Media}

Especially textured and non-crystalline materials show isotropic symmetry. In isotropic symmetry, there are two distinct eigenvalues. The first eigenvalue is of multiplicity one and the other has multiplicity of five. For this symmetry type, equation (2.4) takes the following forms

$$
\begin{gathered}
\left(\hat{c}^{V, \text { Iso }}-\lambda I\right) \hat{N}=0 \\
{\left[\begin{array}{cccccc}
\hat{c}_{11}^{V, I s o}-\lambda & \hat{c}_{12}^{V, \text { Iso }} & \hat{c}_{12}^{V, \text { Iso }} & 0 & 0 & 0 \\
\hat{c}_{12}^{V, \text { Iso }} & \hat{c}_{11}^{V, \text { Iso }}-\lambda & \hat{c}_{12}^{V, \text { Iso }} & 0 & 0 & 0 \\
\hat{c}_{12}^{V, \text { Iso }} & \hat{c}_{12}^{V, I s o} & \hat{c}_{11}^{V, \text { Iso }}-\lambda & 0 & 0 & 0 \\
0 & 0 & 0 & \hat{c}_{44}^{V, I s o}-\lambda & 0 & 0 \\
0 & 0 & 0 & 0 & \hat{c}_{44}^{V, I s o}-\lambda & 0 \\
0 & 0 & 0 & 0 & 0 & \hat{c}_{44}^{V, I s o}-\lambda
\end{array}\right]\left[\begin{array}{c}
\hat{N}_{1} \\
\hat{N}_{2} \\
\hat{N}_{3} \\
\hat{N}_{4} \\
\hat{N}_{5} \\
\hat{N}_{6}
\end{array}\right]=0}
\end{gathered}
$$


where $\hat{C}_{44}^{V, \text { Iso }}=\frac{\left(\hat{c}_{11}^{V, \text { Iso }}-\hat{c}_{12}^{V, \text { Iso }}\right)}{2}$. The elements $\left(\hat{C}^{V, \text { Iso }}\right)$ of matrix can be expressed in terms of the elements ( $\hat{C}^{\text {Tric }}$, elastic stiffnesses) of the triclinic symmetry by using the following terms:

$\hat{c}_{11}^{V, \text { Iso }}=\frac{\left(\hat{c}_{11}^{\text {Tric }}+\hat{c}_{22}^{\text {Tric }}+\hat{c}_{33}^{\text {Tric }}\right)}{3}, \hat{c}_{12}^{V, \text { Iso }}=\frac{\left(\hat{c}_{12}^{\text {Tric }}+\hat{c}_{23}^{\text {Tric }}+\hat{c}_{31}^{\text {Tric }}\right)}{3}, 2 \hat{c}_{44}^{V, \text { Iso }}=\hat{c}_{11}^{V, \text { Iso }}-\hat{c}_{12}^{V, \text { Iso }}$.

The Voigt eigenvalues for isotropic symmetry are

$\lambda_{1}^{V \text {, Iso }}=\hat{c}_{11}^{V \text {, Iso }}+2 \hat{c}_{12}^{V, \text { Iso }}=3 K^{V \text {, Iso }}, \lambda_{(2,3,4,5,6)}^{V \text {, Iso }}=\hat{c}_{11}^{V \text {, Iso }}-\hat{c}_{12}^{V \text {, Iso }}=2 G^{V \text {, Iso }}$.

where $K^{V \text {, Iso }}=\frac{\hat{c}_{11}^{V \text {, Iso }}+2 \hat{c}_{12}^{V, \text { Iso }}}{3}$ (Bulk modulus of Voigt for isotropic symmetry). By using $K^{V \text {, Iso }}$, first linear invariant $I_{1}$ is obtained as

$I_{1}=9 K^{V, \text { Iso }}$

It is also trace of elastic constant matrix for isotropic symmetry. Second invariant is found as

$I_{2}=2 G^{V, \text { Iso }}$

For this symmetry type, equation (2.5) takes the following forms

$$
\begin{gathered}
\left(\hat{s}^{R, I S o}-\left(\frac{1}{\lambda}\right) I\right) \hat{N}=0 \\
{\left[\begin{array}{cccccc}
\hat{s}_{11}^{R, I s o}-\frac{1}{\lambda} & \hat{s}_{12}^{R, I s o} & \hat{s}_{12}^{R, \text { Iso }} & 0 & 0 & 0 \\
\hat{s}_{12}^{R, I s o} & \hat{s}_{11}^{R, I s o}-\frac{1}{\lambda} & \hat{s}_{12}^{R, I s o} & 0 & 0 & 0 \\
\hat{s}_{12}^{R, I S o} & \hat{s}_{12}^{R, I s o} & \hat{s}_{11}^{R, I s o}-\frac{1}{\lambda} & 0 & 0 & 0 \\
0 & 0 & 0 & \hat{s}_{44}^{R, I s o}-\frac{1}{\lambda} & 0 & 0 \\
0 & 0 & 0 & 0 & \hat{s}_{44}^{R, I s o}-\frac{1}{\lambda} & 0 \\
0 & 0 & 0 & 0 & 0 & \hat{s}_{44}^{R, I s o}-\frac{1}{\lambda}
\end{array}\right]\left[\begin{array}{c}
\hat{N}_{1} \\
\hat{N}_{2} \\
\hat{N}_{3} \\
\hat{N}_{4} \\
\hat{N}_{5} \\
\hat{N}_{6}
\end{array}\right]=0}
\end{gathered}
$$

where

$$
\hat{S}_{44}^{R, \text { Iso }}=2\left(\hat{S}_{11}^{R, \text { Iso }}-\hat{S}_{12}^{R, \text { Iso }}\right) .
$$

The elements ( $\hat{s}^{R, I s o}$ ) of matrix can be expressed in terms of the elements ( $\hat{s}^{\text {Tric }}$, elastic compliances) of the triclinic symmetry by using the following terms:

$\hat{s}_{11}^{R, \text { Iso }}=\frac{\left(\hat{s}_{11}^{\text {Tric }}+\hat{s}_{22}^{\text {Tric }}+\hat{s}_{33}^{\text {Tric }}\right)}{3}, \hat{s}_{12}^{R \text { Iso }}=\frac{\left(\hat{s}_{12}^{\text {Tric }}+\hat{s}_{23}^{\text {Tric }}+\hat{s}_{31}^{\text {Tric }}\right)}{3}, \hat{s}_{44}^{R, \text { Iso }}=2\left(\hat{s}_{11}^{R, \text { Iso }}-\hat{s}_{12}^{R, \text { Iso }}\right)$. 
$\frac{1}{\lambda_{1}^{R, I s o}}=\hat{s}_{11}^{R, \text { Iso }}+2 \hat{s}_{12}^{R, \text { Iso }}, \frac{1}{\lambda_{(2,3,4,5,6)}^{R, I s o}}=\hat{s}_{11}^{R, \text { Iso }}-\hat{s}_{12}^{R, \text { Iso }}$.

The generalized Hill inequalities for isotropic symmetry are

$$
\lambda_{1}^{R \text {,Iso }} \leq \lambda_{1}^{\text {eff, Iso }} \leq \lambda_{1}^{V \text {,Iso }} \lambda_{(2,3,4,5,6)}^{R, \text { Iso }} \leq \lambda_{(2,3,4,5,6)}^{\text {eff, Iso }} \leq \lambda_{(2,3,4,5,6)}^{V, \text { Iso }}
$$

\subsection{For Tetragonal Media}

Tetragonal media show tetragonal symmetry. This symmetry type has five distinct eigenvalues which are multiplicity of one, one, one, two and one. For this symmetry type, equation (2.4) takes the following forms

$$
\begin{gathered}
\left(\hat{c}^{V, T e t}-\lambda I\right) \hat{N}=0 \\
{\left[\begin{array}{cccccc}
\hat{c}_{11}^{V, T e t}-\lambda & \hat{c}_{12}^{V, T e t} & \hat{c}_{13}^{V, \text { Tet }} & 0 & 0 & 0 \\
\hat{c}_{12}^{V, T e t} & \hat{c}_{11}^{V, T e t}-\lambda & \hat{c}_{13}^{V, T e t} & 0 & 0 & 0 \\
\hat{c}_{13}^{V, T e t} & \hat{c}_{13}^{V, T e t} & \hat{c}_{33}^{V, T e t}-\lambda & 0 & 0 & 0 \\
0 & 0 & 0 & \hat{c}_{44}^{V, T e t}-\lambda & 0 & 0 \\
0 & 0 & 0 & 0 & \hat{c}_{44}^{V, T e t}-\lambda & 0 \\
0 & 0 & 0 & 0 & 0 & \hat{c}_{66}^{V, T e t}-\lambda
\end{array}\right]\left[\begin{array}{c}
\hat{N}_{1} \\
\hat{N}_{2} \\
\hat{N}_{3} \\
\hat{N}_{4} \\
\hat{N}_{5} \\
\hat{N}_{6}
\end{array}\right]=0}
\end{gathered}
$$

The elements ( $\hat{c}^{V, T e t}$ ) of matrix can be expressed in terms of the elements ( $\hat{c}^{\text {Tric }}$, elastic stiffnesses) of the triclinic symmetry by using the following terms:

$$
\begin{aligned}
& \hat{c}_{11}^{V, \text { Tet }}=\frac{\left(\hat{c}_{11}^{\text {Tric }}+\hat{c}_{22}^{\text {Tric }}\right)}{2}, \hat{c}_{12}^{V, \text { Tet }}=\hat{c}_{12}^{\text {Tric }}, \hat{c}_{13}^{V, \text { Tet }}=\frac{\left(\hat{c}_{13}^{\text {Tric }}+\hat{c}_{23}^{\text {Tric }}\right)}{2}, \hat{c}_{33}^{V, \text { Tet }}=\hat{c}_{33}^{\text {Tric }}, \hat{c}_{44}^{V, \text { Tet }}=\frac{\left(\hat{c}_{44}^{\text {Tric }}+\hat{c}_{55}^{\text {Tric }}\right)}{2}, \\
& \hat{c}_{66}^{V, \text { Tet }}=\hat{c}_{66}^{\text {Tric }} .
\end{aligned}
$$

The Voigt eigenvalues for tetragonal symmetry are

$$
\begin{aligned}
& \lambda_{1}^{V, \text { Tet }}=\frac{1}{2}\left[\hat{c}_{11}^{V, \text { Tet }}+\hat{c}_{12}^{V, \text { Tet }}+\hat{c}_{33}^{V, \text { Tet }}+\sqrt{8\left(\hat{c}_{13}^{V, \text { Tet }}\right)^{2}+\left(\hat{c}_{11}^{V, \text { Tet }}+\hat{c}_{12}^{V, \text { Tet }}-\hat{c}_{33}^{V, \text { Tet }}\right)^{2}}\right], \\
& \lambda_{2}^{V, \text { Tet }}=\frac{1}{2}\left[\hat{c}_{11}^{V, \text { Tet }}+\hat{c}_{12}^{V, \text { Tet }}+\hat{c}_{33}^{V, \text { Tet }}-\sqrt{8\left(\hat{c}_{13}^{V, \text { Tet }}\right)^{2}+\left(\hat{c}_{11}^{V, \text { Tet }}+\hat{c}_{12}^{V, \text { Tet }}-\hat{c}_{33}^{V, \text { Tet }}\right)^{2}}\right], \\
& \lambda_{3}^{V, \text { Tet }}=\hat{c}_{11}^{V, \text { Tet }}-\hat{c}_{12}^{V, \text { Tet }}, \lambda_{(4,5)}^{V, \text { Tet }}=2 \hat{c}_{44}^{V, \text { Tet }}, \lambda_{6}^{V, \text { Tet }}=2 \hat{c}_{66}^{V, \text { Tet }} .
\end{aligned}
$$

For this symmetry type, equation (2.5) takes the following forms

$$
\left(\hat{s}^{R, T e t}-\left(\frac{1}{\lambda}\right) I\right) \hat{N}=0
$$




$\left[\begin{array}{cccccc}\hat{s}_{11}^{R, \text { Tet }}-\frac{1}{\lambda} & \hat{s}_{12}^{R, \text { Tet }} & \hat{s}_{13}^{R, \text { Tet }} & 0 & 0 & 0 \\ \hat{s}_{12}^{R, \text { Tet }} & \hat{s}_{11}^{R, \text { Tet }}-\frac{1}{\lambda} & \hat{s}_{13}^{R, \text { Tet }} & 0 & 0 & 0 \\ \hat{s}_{13}^{R, \text { Tet }} & \hat{s}_{13}^{R, \text { Tet }} & \hat{s}_{33}^{R, \text { Tet }}-\frac{1}{\lambda} & 0 & 0 & 0 \\ 0 & 0 & 0 & \hat{s}_{44}^{R, \text { Tet }}-\frac{1}{\lambda} & 0 & 0 \\ 0 & 0 & 0 & 0 & \hat{s}_{44}^{R, \text { Tet }}-\frac{1}{\lambda} & 0 \\ 0 & 0 & 0 & 0 & 0 & \hat{s}_{66}^{R, \text { Tet }}-\frac{1}{\lambda}\end{array}\right]\left[\begin{array}{l}\hat{N}_{1} \\ \hat{N}_{2} \\ \hat{N}_{3} \\ \hat{N}_{4} \\ \hat{N}_{5} \\ \hat{N}_{6}\end{array}\right]=0$

The Reuss eigenvalues for tetragonal symmetry are $\lambda_{1}^{R, \text { Tet }}=\frac{\frac{1}{2}}{\left(\hat{s}_{11}^{R, \text { Tet }} \hat{s}_{33}^{R, \text { Tet }}+\hat{s}_{12}^{R, \text { Tet }} \hat{s}_{33}^{R, \text { Tet }}-2\left(\hat{s}_{13}^{R, \text { Tet }}\right)^{2}\right.}(a+\sqrt{b})$, $\lambda_{2}^{R, \text { Tet }}=\frac{\frac{1}{2}}{\left(\left(\hat{s}_{11}^{R, \text { Tet }} \hat{S}_{33}^{R, \text { Tet }}+\hat{s}_{12}^{R, \text { Tet }} \hat{S}_{33}^{R, \text { Tet }}-2\left(\hat{s}_{13}^{R, \text { Tet }}\right)^{2}\right.\right.}(a-\sqrt{b})$,

where $a=\hat{s}_{33}^{R, T e t}+\hat{s}_{12}^{R, T e t}+\hat{s}_{11}^{R, T e t}$

and $b=\left(\left(\hat{S}_{33}^{R, T e t}\right)^{2}-2 \hat{s}_{12}^{R, T e t} \hat{S}_{33}^{R, T e t}-2 \hat{S}_{11}^{R, T e t} \hat{S}_{33}^{R, T e t}+\left(\hat{S}_{12}^{R, T e t}\right)^{2}+2 \hat{s}_{11}^{R, T e t} \hat{S}_{12}^{R, T e t}+\left(\hat{S}_{11}^{R, T e t}\right)^{2}+8\left(\hat{S}_{13}^{R, T e t}\right)^{2}\right)$.

$\lambda_{3}^{R, \text { Tet }}=\frac{1}{\hat{S}_{11}^{R, \text { Tet }}-\hat{s}_{12}^{R, \text { Tet }}}, \lambda_{(4,5)}^{R, \text { Tet }}=\frac{2}{\hat{s}_{44}^{R, \text { Tet }}}, \lambda_{6}^{R, \text { Tet }}=\frac{2}{\hat{s}_{66}^{R, \text { Tet }}}$.

The generalized Hill inequalities for tetragonal symmetry are

$$
\begin{array}{ll}
\lambda_{1}^{R, \text { Tet }} \leq \lambda_{1}^{e f f, \text { Tet }} \leq \lambda_{1}^{V, \text { Tet }}, & \lambda_{2}^{R, \text { Tet }} \leq \lambda_{2}^{e f f, \text { Tet }} \leq \lambda_{2}^{V, \text { Tet }}, \quad \lambda_{3}^{R, \text { Tet }} \leq \lambda_{3}^{\text {eff, Tet }} \leq \lambda_{3}^{V, \text { Tet }}, \\
\lambda_{(4,5)}^{R, \text { Tet }} \leq \lambda_{(4,5)}^{e f f, \text { Tet }} \leq \lambda_{(4,5)}^{V, \text { Tet }}, \quad \lambda_{6}^{R, \text { Tet }} \leq \lambda_{6}^{e f f, \text { Tet }} \leq \lambda_{6}^{V, \text { Tet }} .
\end{array}
$$

\subsection{For Transversely Isotropic Media}

Most textured and non-crystalline materials exhibit transversely isotropic symmetry. Transversely isotropic medium is a special case of hexagonal symmetry. The elastic constant tensor of transversely isotropic symmetry has almost the same structure as that of hexagonal symmetry therefore the two symmetries can be considered together. Transversely isotropic symmetry has four distinct eigenvalues with multiplicity of one, one, two and two. For this symmetry type, equation (2.4) takes the following forms

$$
\left(\hat{c}^{V, T \text { Trans }}-\lambda I\right) \hat{N}=0
$$


$\left[\begin{array}{cccccc}\hat{c}_{11}^{V, \text { Trans }}-\lambda & \hat{c}_{12}^{V, \text { Trans }} & \hat{c}_{13}^{V, \text { Trans }} & 0 & 0 & 0 \\ \hat{c}_{12}^{V, \text { Trans }} & \hat{c}_{11}^{V, \text { Trans }}-\lambda & \hat{c}_{13}^{V, \text { Trans }} & 0 & 0 & 0 \\ \hat{c}_{13}^{V, \text { Trans }} & \hat{c}_{13}^{V, \text { Trans }} & \hat{c}_{33}^{V, \text { Trans }}-\lambda & 0 & 0 & 0 \\ 0 & 0 & 0 & \hat{c}_{44}^{V, \text { Trans }}-\lambda & 0 & 0 \\ 0 & 0 & 0 & 0 & \hat{c}_{44}^{V, \text { Trans }}-\lambda & 0 \\ 0 & 0 & 0 & 0 & 0 & \hat{c}_{66}^{V, \text { Trans }}-\lambda\end{array}\right]\left[\begin{array}{c}\hat{N}_{1} \\ \hat{N}_{2} \\ \hat{N}_{3} \\ \hat{N}_{4} \\ \hat{N}_{5} \\ \hat{N}_{6}\end{array}\right]=0$

where $\hat{c}_{66}^{V, \text { Trans }}=\frac{\left(\hat{c}_{11}^{V, \text { Trans }}-\hat{c}_{12}^{V, \text { Trans }}\right)}{2}$.

The elements ( $\hat{c}^{V, \text { Trans }}$ ) of matrix can be expressed in terms of the elements ( $\hat{c}^{\text {Tric }}$, elastic stiffnesses) of the triclinic symmetry by using the following terms: $\hat{c}_{11}^{V \text {,Trans }}=\frac{1}{2}\left(\hat{c}_{11}^{\text {Tric }}+\hat{c}_{22}^{\text {Tric }}\right), \hat{c}_{12}^{V \text {,Trans }}=\hat{c}_{12}^{\text {Tric }}, \hat{c}_{13}^{V, \text { Trans }}=\frac{1}{2}\left(\hat{c}_{13}^{\text {Tric }}+\hat{c}_{23}^{\text {Tric }}\right), \hat{c}_{33}^{V \text {,Trans }}=\hat{c}_{33}^{\text {Tric }}$

$\hat{C}_{44}^{V, T r a n s}=\frac{\left(\hat{C}_{44}^{T}+\hat{C}_{55}^{T}\right)}{2}$.

The Voigt eigenvalues for transversely isotropic symmetry are

$$
\begin{aligned}
& \lambda_{1}^{V, \text { Trans }}=\frac{1}{2}\left[\hat{c}_{11}^{V, \text { Trans }}+\hat{c}_{12}^{V, \text { Trans }}+\hat{c}_{33}^{V, \text { Trans }}+\sqrt{8\left(\hat{c}_{13}^{V, \text { Trans }}\right)^{2}+\left(\hat{c}_{11}^{V, \text { Trans }}+\hat{c}_{12}^{V, \text { Trans }}-\hat{c}_{33}^{V, \text { Trans }}\right)^{2}}\right], \\
& \lambda_{2}^{V, \text { Trans }}=\frac{1}{2}\left[\hat{c}_{11}^{V, \text { Trans }}+\hat{c}_{12}^{V, \text { Trans }}+\hat{c}_{33}^{V, \text { Trans }}-\sqrt{8\left(\hat{c}_{13}^{V, \text { Trans }}\right)^{2}+\left(\hat{c}_{11}^{V, \text { Trans }}+\hat{c}_{12}^{V, \text { Trans }}-\hat{c}_{33}^{V, \text { Trans }}\right)^{2}}\right], \\
& \lambda_{(3,6)}^{V, \text { Trans }}=\hat{c}_{11}^{V, \text { Trans }}-\hat{c}_{12}^{V, \text { Trans }}, \lambda_{(4,5)}^{V, \text { Trans }}=2 \hat{c}_{44}^{V, \text { Trans }} .
\end{aligned}
$$

For this symmetry type, equation (2.5) takes the following forms

$$
\begin{aligned}
& \left(\hat{S}^{R, \text { Trans }}-\left(\frac{1}{\lambda}\right) I\right) \hat{N}=0 \\
& {\left[\begin{array}{cccccc}
\hat{s}_{11}^{R, \text { Trans }}-\frac{1}{\lambda} & \hat{s}_{12}^{R, \text { Trans }} & \hat{s}_{13}^{R, \text { Trans }} & 0 & 0 & 0 \\
\hat{s}_{12}^{R, \text { Trans }} & \hat{s}_{11}^{R, \text { Trans }}-\frac{1}{\lambda} & \hat{s}_{13}^{R, \text { Trans }} & 0 & 0 & 0 \\
\hat{s}_{13}^{R, \text { Trans }} & \hat{s}_{13}^{R, \text { Trans }} & \hat{s}_{33}^{R, \text { Trans }}-\frac{1}{\lambda} & 0 & 0 & 0 \\
0 & 0 & 0 & \hat{s}_{44}^{R, \text { Trans }}-\frac{1}{\lambda} & 0 & 0 \\
0 & 0 & 0 & 0 & \hat{s}_{44}^{R, \text { Trans }}-\frac{1}{\lambda} & 0 \\
0 & 0 & 0 & 0 & 0 & \hat{s}_{66}^{R, \text { Trans }}-\frac{1}{\lambda}
\end{array}\right]\left[\begin{array}{c}
\hat{N}_{1} \\
\hat{N}_{2} \\
\hat{N}_{3} \\
\hat{N}_{4} \\
\hat{N}_{5} \\
\hat{N}_{6}
\end{array}\right]=0(4.4 .8)}
\end{aligned}
$$

where $\quad \hat{S}_{66}^{R, \text { Trans }}=2\left(\hat{s}_{11}^{R \text {,Trans }}-\hat{s}_{12}^{R, \text { Trans }}\right)$.

The Reuss eigenvalues for transversely isotropic symmetry are 


$$
\begin{aligned}
& \lambda_{1}^{R, \text { Trans }}=\frac{\frac{1}{2}}{\hat{s}_{11}^{R, \text { Trans }} \hat{s}_{33}^{R, \text { Trans }}+\hat{s}_{12}^{R, \text { Trans }} \hat{s}_{33}^{R, \text { Trans }}-2\left(\hat{s}_{13}^{R, \text { Trans }}\right)^{2}}(c+\sqrt{d}), \\
& \lambda_{2}^{R, \text { Trans }}=\frac{\frac{1}{2}}{\hat{s}_{11}^{R, \text { Trans }} \hat{s}_{33}^{R, \text { Trans }}+\hat{s}_{12}^{R, \text { Trans }} \hat{s}_{33}^{R, \text { Trans }}-2\left(\hat{s}_{13}^{R, \text { Trans }}\right)^{2}}(c-\sqrt{d}),
\end{aligned}
$$

where $c=\hat{s}_{33}^{R, \text { Trans }}+\hat{s}_{12}^{R \text {,Trans }}+\hat{s}_{11}^{R, \text { Trans }}$ and

$$
\begin{aligned}
& d=\left(\left(\hat{s}_{33}^{R, \text { Trans }}\right)^{2}-2 \hat{s}_{12}^{R, \text { Trans }} \hat{S}_{33}^{R, \text { Trans }}-2 \hat{s}_{11}^{R, \text { Trans }} \hat{s}_{33}^{R, \text { Trans }}+\left(\hat{S}_{12}^{R, \text { Trans }}\right)^{2}+2 \hat{s}_{11}^{R, \text { Trans }} \hat{s}_{12}^{R, \text { Trans }}+\left(\hat{s}_{11}^{R, \text { Trans }}\right)^{2}+8\left(\hat{s}_{13}^{R, \text { Trans }}\right)^{2}\right) . \\
& \lambda_{(3,6)}^{R, \text { Trans }}=\frac{1}{\hat{s}_{11}^{R, \text { Trans }}-\hat{s}_{12}^{R, \text { Trans }}}, \lambda_{(4,5)}^{R, \text { Trans }}=\frac{2}{\hat{s}_{44}^{R, \text { Trans }}} .
\end{aligned}
$$

The generalized Hill inequalities for transversely isotropic symmetry are

$$
\begin{array}{ll}
\lambda_{1}^{R, \text { Trans }} \leq \lambda_{1}^{\text {eff, Trans }} \leq \lambda_{1}^{V, \text { Trans }}, & \lambda_{2}^{R, \text { Trans }} \leq \lambda_{2}^{\text {eff, Trans }} \leq \lambda_{2}^{V, \text { Trans },} \quad \lambda_{(3,6)}^{R, \text { Trans }} \leq \lambda_{(3,6)}^{\text {eff, Trans }} \leq \lambda_{(3,6)}^{V, \text { Trans }}, \\
\lambda_{(4,5)}^{R, \text { Trans }} \leq \lambda_{(4,5)}^{\text {eff, Trans }} \leq \lambda_{(4,5)}^{V, \text { Trans }} .
\end{array}
$$

\subsection{For Trigonal Media}

Trigonal media exhibit trigonal symmetry. Trigonal media have 4 distinct eigenvalues with multiplicity of one, one, two and two. For this symmetry type, equation (2.4) takes the following forms

$$
\left(\hat{C}^{V, T r i g}-\lambda I\right) \hat{N}=0
$$

$\left[\begin{array}{cccccc}\hat{c}_{11}^{V, \text { Trig }}-\lambda & \hat{c}_{12}^{V, \text { Trig }} & \hat{c}_{13}^{V, \text { Trig }} & \hat{c}_{14}^{V, \text { Trig }} & 0 & 0 \\ \hat{c}_{12}^{V, \text { Trig }} & \hat{c}_{22}^{V, \text { Trig }}-\lambda & \hat{c}_{23}^{V, \text { Trig }} & -\hat{c}_{14}^{V, \text { Trig }} & 0 & 0 \\ \hat{c}_{13}^{V, \text { Trig }} & \hat{c}_{23}^{V, \text { Trig }} & \hat{c}_{33}^{V, \text { Trig }}-\lambda & 0 & 0 & 0 \\ \hat{c}_{14}^{V, \text { Trig }} & -\hat{c}_{14}^{V, \text { Trig }} & 0 & \hat{c}_{44}^{V, \text { Trig }}-\lambda & 0 & 0 \\ 0 & 0 & 0 & 0 & \hat{c}_{55}^{V, \text { Trig }}-\lambda & \hat{c}_{14}^{V, \text { Trig }} \\ 0 & 0 & 0 & 0 & \hat{c}_{14}^{V, \text { Trig }} & \hat{c}_{66}^{V, \text { Trig }}-\lambda\end{array}\right]\left[\begin{array}{c}\hat{N}_{1} \\ \hat{N}_{2} \\ \hat{N}_{3} \\ \hat{N}_{4} \\ \hat{N}_{5} \\ \hat{N}_{6}\end{array}\right]=0$

where $\hat{c}_{66}^{V, \text { Trig }}=\frac{\left(\hat{c}_{11}^{V, T r i g}-\hat{c}_{12}^{V, T r i g}\right)}{2}$.

The elements ( $\hat{c}^{V, T r i g}$ ) of matrix can be expressed in terms of the elements( $\hat{c}^{\text {Tric }}$, elastic stiffnesses) of the triclinic symmetry by using the following relations:

$\hat{c}_{11}^{V, \text { Trig }}=\frac{1}{8}\left(3 \hat{c}_{11}^{\text {Tric }}+2 \hat{c}_{12}^{\text {Tric }}+3 \hat{c}_{22}^{\text {Tric }}+2 \hat{c}_{66}^{\text {Tric }}\right), \hat{c}_{12}^{V, \text { Trig }}=\frac{1}{8}\left(\hat{c}_{11}^{\text {Tric }}+6 \hat{c}_{12}^{\text {Tric }}+\hat{c}_{22}^{\text {Tric }}-2 \hat{c}_{66}^{\text {Tric }}\right), \hat{c}_{13}^{V, \text { Trig }}=\frac{1}{2}\left(\hat{c}_{13}^{\text {Tric }}+\hat{c}_{23}^{\text {Tric }}\right)$, 
$\hat{c}_{14}^{V, \text { Trig }}=\frac{1}{4}\left(\hat{c}_{14}^{\text {Tric }}-\hat{c}_{24}^{\text {Tric }}+\sqrt{2} \hat{c}_{56}^{\text {Tric }}\right), \hat{c}_{33}^{V, \text { Trig }}=\hat{c}_{33}^{\text {Tric }}, \hat{c}_{44}^{V, \text { Trig }}=\frac{1}{2}\left(\hat{c}_{44}^{\text {Tric }}+\hat{c}_{55}^{\text {Tric }}\right)$.

The Voigt eigenvalues for trigonal symmetry are

$$
\begin{aligned}
& \lambda_{1}^{V, \text { Trig }}=\frac{1}{2}\left[\hat{c}_{11}^{V, \text { Trig }}+\hat{c}_{12}^{V, \text { Trig }}+\hat{c}_{33}^{V, \text { Trig }}+\sqrt{8\left(\hat{c}_{13}^{V, \text { Trig }}\right)^{2}+\left(\hat{c}_{11}^{V, \text { Trig }}+\hat{c}_{12}^{V, \text { Trig }}-\hat{c}_{33}^{V, \text { Trig }}\right)^{2}}\right], \\
& \lambda_{2}^{V, \text { Trig }}=\frac{1}{2}\left[\hat{c}_{11}^{V, \text { Trig }}+\hat{c}_{12}^{V, \text { Trig }}+\hat{c}_{33}^{V, \text { Trig }}-\sqrt{8\left(\hat{c}_{13}^{V, \text { Trig }}\right)^{2}+\left(\hat{c}_{11}^{V, \text { Trig }}+\hat{c}_{12}^{V, \text { Trig }}-\hat{c}_{33}^{V, \text { Trig }}\right)^{2}}\right], \\
& \lambda_{(3,6)}^{V, \text { Trig }}=\frac{1}{2}\left[\hat{c}_{11}^{V, \text { Trig }}-\hat{c}_{12}^{V, \text { Trig }}+\hat{c}_{44}^{V, \text { Trig }}+\sqrt{8\left(\hat{c}_{14}^{V, \text { Trig }}\right)^{2}+\left(\hat{c}_{11}^{V, \text { Trig }}-\hat{c}_{12}^{V, \text { Trig }}-\hat{c}_{44}^{V, \text { Trig }}\right)^{2}}\right], \\
& \lambda_{(4,5)}^{V, \text { Trig }}=\frac{1}{2}\left[\hat{c}_{11}^{V, \text { Trig }}-\hat{c}_{12}^{V, \text { Trig }}+\hat{c}_{44}^{V, \text { Trig }}-\sqrt{8\left(\hat{c}_{14}^{V, \text { Trig }}\right)^{2}+\left(\hat{c}_{11}^{V, \text { Trig }}-\hat{c}_{12}^{V, \text { Trig }}-\hat{c}_{44}^{V, \text { Trig }}\right)^{2}}\right],
\end{aligned}
$$

For this symmetry type, equation (2.5) takes the following forms

$$
\left(\hat{s}^{R, T r i g}-\left(\frac{1}{\lambda}\right) I\right) \hat{N}=0
$$

$\left[\begin{array}{cccccc}\hat{s}_{11}^{R, \text { Trig }}-\frac{1}{\lambda} & \hat{s}_{12}^{R, \text { Trig }} & \hat{s}_{13}^{R, \text { Trig }} & \hat{s}_{14}^{R, \text { Trig }} & 0 & 0 \\ \hat{s}_{12}^{R, \text { Trig }} & \hat{s}_{22}^{R, \text { Trig }}-\frac{1}{\lambda} & \hat{s}_{23}^{R, \text { Trig }} & -\hat{s}_{14}^{R, \text { Trig }} & 0 & 0 \\ \hat{s}_{13}^{R, \text { Trig }} & \hat{s}_{23}^{R, \text { Trig }} & \hat{s}_{33}^{R, \text { Trig }}-\frac{1}{\lambda} & 0 & 0 & 0 \\ \hat{s}_{14}^{R, \text { Trig }} & -\hat{s}_{14}^{R, \text { Trig }} & 0 & \hat{s}_{44}^{R, \text { Trig }}-\frac{1}{\lambda} & 0 & 0 \\ 0 & 0 & 0 & 0 & \hat{s}_{55}^{R, \text { Trig }}-\frac{1}{\lambda} & \hat{s}_{14}^{R, \text { Trig }} \\ 0 & 0 & 0 & 0 & \hat{s}_{14}^{R, \text { Trig }} & \hat{s}_{66}^{R, \text { Trig }}-\frac{1}{\lambda}\end{array}\right]\left[\begin{array}{c}\hat{N}_{1} \\ \hat{N}_{2} \\ \hat{N}_{3} \\ \hat{N}_{4} \\ \hat{N}_{5} \\ \hat{N}_{6}\end{array}\right]=0$ (4.5.10)

where $\hat{s}_{66}^{R, \text { Trig }}=2\left(\hat{s}_{11}^{R, \text { Trig }}-\hat{s}_{12}^{R, \text { Trig }}\right)$.

The Reuss eigenvalues for trigonal symmetry

$$
\begin{aligned}
& \text { are } \lambda_{1}^{R, \text { Trig }}=\frac{\frac{1}{2}}{\left(\hat{s}_{11}^{R, \text { Trig }} \hat{s}_{33}^{R, \text { Trig }}+\hat{s}_{12}^{\text {R,Trig }} \hat{s}_{33}^{\text {,Trig }}-2\left(\hat{s}_{13}^{\text {,Trig }}\right)^{2}\right)}(e+\sqrt{f}), \\
& \lambda_{2}^{R, \text { Trig }}=\frac{\frac{1}{2}}{\left(\hat{s}_{11}^{\text {R,Trig }} \hat{s}_{33}^{\text {,Trig }}+\hat{s}_{12}^{\text {,Trig }} \hat{s}_{33}^{\text {R,Trig }}-2\left(\hat{s}_{13}^{\text {R,Trig }}\right)^{2}\right)}(e-\sqrt{f})
\end{aligned}
$$

where $e=\hat{s}_{33}^{R, \text { Trig }}+\hat{s}_{12}^{R, \text { Trig }}+\hat{s}_{11}^{R, \text { Trig }}$, and $f=\left(\hat{s}_{33}^{R, \text { Trig }}\right)^{2}-2 \hat{s}_{12}^{R, \text { Trig }} \hat{s}_{33}^{R, \text { Trig }}-2 \hat{s}_{11}^{R, \text { Trig }} \hat{s}_{33}^{R, \text { Trig }}+\left(\left(\hat{s}_{12}^{R, \text { Trig }}\right)^{2}+2 \hat{s}_{11}^{R, \text { Trig }} \hat{s}_{12}^{R, \text { Trig }}+\left(\hat{s}_{11}^{R, \text { Trig }}\right)^{2}+8\left(\hat{s}_{13}^{R, \text { Trig }}\right)^{2}\right.$. 


$$
\begin{aligned}
& \lambda_{(3,6)}^{R, \text { Trig }}=\frac{\frac{1}{2}}{\left(2\left(\hat{s}_{14}^{R, \text { Trig }}\right)^{2}-2 \hat{s}_{44}^{R, \text { Trig }} \hat{s}_{11}^{R, \text { Trig }}+2 \hat{s}_{44}^{R, \text { Trig }} \hat{s}_{12}^{R, \text { Trig }}\right)}(g-\sqrt{h}), \\
& \lambda_{(4,5)}^{R, \text { Trig }}=\frac{\frac{1}{2}}{\left(2\left(\hat{s}_{14}^{R, \text { Trig }}\right)^{2}-2 \hat{s}_{44}^{R, \text { Trig }} \hat{s}_{11}^{R, \text { Trig }}+2 \hat{s}_{44}^{R, \text { Trig }} \hat{s}_{12}^{R, \text { Trig }}\right)}(g+\sqrt{h}) .
\end{aligned}
$$

Where $g=\hat{s}_{12}^{R, \text { Trig }}-\hat{s}_{11}^{R, \text { Trig }}-2 \hat{s}_{44}^{R, \text { Trig }}$, and

$$
h=\left(\hat{S}_{11}^{R, \text { Trig }}\right)^{2}-2 \hat{S}_{12}^{R, \text { Trig }} \hat{S}_{11}^{R, \text { Trig }}-4 \hat{S}_{44}^{R, \text { Trig }} \hat{S}_{11}^{R, \text { Trig }}+\left(\hat{S}_{12}^{R, \text { Trig }}\right)^{2}+4 \hat{s}_{44}^{R, \text { Trig }} \hat{S}_{12}^{R, \text { Trig }}+4\left(\hat{S}_{44}^{R, \text { Trig }}\right)^{2}+8\left(\hat{s}_{14}^{R, \text { Trig }}\right)^{2} .
$$

The generalized Hill inequalities for trigonal symmetry are

$$
\lambda_{1}^{R, \text { Trig }} \leq \lambda_{1}^{\text {eff,Trig }} \leq \lambda_{1}^{V, \text { Trig }}, \quad \lambda_{2}^{R, \text { Trig }} \leq \lambda_{2}^{\text {eff, Trig }} \leq \lambda_{2}^{V, \text { Trig }}, \quad \lambda_{(3,6)}^{R, \text { Trig }} \leq \lambda_{(3,6)}^{\text {eff, Trig }} \leq \lambda_{(3,6)}^{V, \text { Trig }}, \quad \lambda_{(4,5)}^{R, \text { Trig }} \leq \lambda_{(4,5)}^{\text {eff ,Trig }} \leq \lambda_{(4,5)}^{V, \text { Trig }}
$$

\subsection{For Orthorhombic Media}

Orthorhombic media show an orthorhombic symmetry. When the same symmetry is applied to textured or non-crystalline materials, it is generally called orthotropy. However, for most practical purposes, orthotropic symmetry is equivalent to orthorhombic symmetry. There are six distinct eigenvalues with multiplicity one in orthorhombic symmetry. The elements ( $\hat{c}^{V, \text { Ort }}$ ) of elastic stiffness matrix can be expressed in terms of the elements( $\hat{c}^{\text {Tric }}$, elastic stiffnesses) of the triclinic symmetry by the following matrix:

$$
\hat{c}^{V, \text { Ort }}=\left[\begin{array}{cccccc}
\hat{c}_{11}^{\text {Tric }} & \hat{c}_{12}^{\text {Tric }} & \hat{c}_{13}^{\text {Tric }} & 0 & 0 & 0 \\
\hat{c}_{12}^{\text {Tric }} & \hat{c}_{22}^{\text {Tric }} & \hat{c}_{23}^{\text {Tric }} & 0 & 0 & 0 \\
\hat{c}_{13}^{\text {Tric }} & \hat{c}_{23}^{\text {Tric }} & \hat{c}_{33}^{\text {Tric }} & 0 & 0 & 0 \\
0 & 0 & 0 & \hat{c}_{44}^{\text {Tric }} & 0 & 0 \\
0 & 0 & 0 & 0 & \hat{c}_{55}^{\text {Tric }} & 0 \\
0 & 0 & 0 & 0 & 0 & \hat{c}_{66}^{\text {Tric }}
\end{array}\right]
$$

For this symmetry type, equation (2.4) takes the following forms

$$
\left(\hat{c}^{V, \text { Ort }}-\lambda I\right) \hat{N}=0
$$

$$
\left[\begin{array}{cccccc}
\hat{c}_{11}^{V, \text { Ort }}-\lambda & \hat{c}_{12}^{V, \text { Ort }} & \hat{c}_{13}^{V, \text { Ort }} & 0 & 0 & 0 \\
\hat{c}_{12}^{V, \text { Ort }} & \hat{c}_{22}^{V, \text { Ort }}-\lambda & \hat{c}_{23}^{V, \text { Ort }} & 0 & 0 & 0 \\
\hat{c}_{13}^{V, \text { Ort }} & \hat{c}_{23}^{V, \text { Ort }} & \hat{c}_{33}^{V, \text { Ort }}-\lambda & 0 & 0 & 0 \\
0 & 0 & 0 & \hat{c}_{44}^{V, \text { Ort }}-\lambda & 0 & 0 \\
0 & 0 & 0 & 0 & \hat{c}_{55}^{V, \text { Ort }}-\lambda & 0 \\
0 & 0 & 0 & 0 & 0 & \hat{c}_{66}^{V, \text { Ort }}-\lambda
\end{array}\right]\left[\begin{array}{c}
\hat{N}_{1} \\
\hat{N}_{2} \\
\hat{N}_{3} \\
\hat{N}_{4} \\
\hat{N}_{5} \\
\hat{N}_{6}
\end{array}\right]=0
$$


The Voigt eigenvalues of orthorhombic media can be obtained from above matrix. The elements ( $\hat{S}^{R \text {, Ort }}$ ) of matrix can be expressed in terms of the elements ( $\hat{s}^{\text {Tric }}$, elastic compliances) of the triclinic symmetry by the following matrix:

$$
\hat{S}^{R, \text { Ort }}=\left[\begin{array}{cccccc}
\hat{s}_{11}^{\text {Tric }} & \hat{s}_{12}^{\text {Tric }} & \hat{s}_{13}^{\text {Tric }} & 0 & 0 & 0 \\
\hat{s}_{12}^{\text {Tric }} & \hat{s}_{22}^{\text {Tric }} & \hat{s}_{23}^{\text {Tric }} & 0 & 0 & 0 \\
\hat{s}_{13}^{\text {Tric }} & \hat{s}_{23}^{\text {Tric }} & \hat{s}_{33}^{\text {Tric }} & 0 & 0 & 0 \\
0 & 0 & 0 & \hat{s}_{44}^{\text {Tric }} & 0 & 0 \\
0 & 0 & 0 & 0 & \hat{s}_{55}^{\text {Tric }} & 0 \\
0 & 0 & 0 & 0 & 0 & \hat{s}_{66}^{\text {Tric }}
\end{array}\right]
$$

For this symmetry type, equation (2.5) takes the following forms

$$
\begin{gathered}
\left(\hat{s}^{R, \text { Ort }}-\left(\frac{1}{\lambda}\right) I\right) \hat{N}=0 \\
{\left[\begin{array}{cccccc}
\hat{s}_{11}^{R, \text { Ort }}-\frac{1}{\lambda} & \hat{s}_{12}^{R, \text { Ort }} & \hat{s}_{13}^{R, \text { Ort }} & 0 & 0 & 0 \\
\hat{s}_{12}^{R, \text { Ort }} & \hat{s}_{22}^{R, \text { Ort }}-\frac{1}{\lambda} & \hat{s}_{23}^{R, \text { Ort }} & 0 & 0 & 0 \\
\hat{s}_{13}^{R, \text { Ort }} & \hat{s}_{23}^{R, \text { Ort }} & \hat{s}_{33}^{R, \text { Ort }}-\frac{1}{\lambda} & 0 & 0 & 0 \\
0 & 0 & 0 & \hat{s}_{44}^{R, \text { Ort }}-\frac{1}{\lambda} & 0 & 0 \\
0 & 0 & 0 & 0 & \hat{s}_{55}^{R, \text { Ort }}-\frac{1}{\lambda} & 0 \\
0 & 0 & 0 & 0 & 0 & \hat{s}_{66}^{R, \text { Ort }}-\frac{1}{\lambda}
\end{array}\right]\left[\begin{array}{c}
\hat{N}_{1} \\
\hat{N}_{2} \\
\hat{N}_{3} \\
\hat{N}_{4} \\
\hat{N}_{5} \\
\hat{N}_{6}
\end{array}\right]=0}
\end{gathered}
$$

The Reuss eigenvalues for orthorhombic symmetry can be obtained from the above matrix.

The generalized Hill inequalities for orthorhombic symmetry are

$$
\begin{array}{ll}
\lambda_{1}^{R, \text { Ort }} \leq \lambda_{1}^{\text {eff, Ort }} \leq \lambda_{1}^{V, \text { Ort }}, & \lambda_{2}^{R, \text { Ort }} \leq \lambda_{2}^{\text {eff, Ort }} \leq \lambda_{2}^{V, \text { Ort }}, \quad \lambda_{3}^{R, \text { Ort }} \leq \lambda_{3}^{\text {eff, Ort }} \leq \lambda_{3}^{V, \text { Ort }}, \\
\lambda_{4}^{R, \text { Ort }} \leq \lambda_{4}^{\text {eff, Ort }} \leq \lambda_{4}^{V, \text { Ort }}, \quad \lambda_{5}^{R, \text { Ort }} \leq \lambda_{5}^{\text {eff, Ort }} \leq \lambda_{5}^{V, \text { Ort }}, \quad \lambda_{6}^{R, \text { Ort }} \leq \lambda_{6}^{\text {eff, Ort }} \leq \lambda_{6}^{V, \text { Ort }} .
\end{array}
$$

\subsection{For Monoclinic Media}

Monoclinic media show monoclinic symmetry. Monoclinic symmetry has six distinct eigenvalues with multiplicity of one. The elements ( $\hat{C}^{V, \text { Mon }}$ ) of matrix can be expressed in terms of the elements( $\hat{C}^{\text {Tric }}$, elastic stiffnesses) of the triclinic symmetry by the following matrix: 


$$
\hat{c}^{V, \text { Mon }}=\left[\begin{array}{cccccc}
\hat{c}_{11}^{\text {Tric }} & \hat{c}_{12}^{\text {Tric }} & \hat{c}_{13}^{\text {Tric }} & 0 & \hat{c}_{15}^{\text {Tric }} & 0 \\
\hat{c}_{12}^{\text {Tric }} & \hat{c}_{22}^{\text {Tric }} & \hat{c}_{23}^{\text {Tric }} & 0 & \hat{c}_{25}^{\text {Tric }} & 0 \\
\hat{c}_{13}^{\text {Tric }} & \hat{c}_{23}^{\text {Tric }} & \hat{c}_{33}^{\text {Tric }} & 0 & \hat{c}_{35}^{\text {Tric }} & 0 \\
0 & 0 & 0 & \hat{c}_{44}^{\text {Tric }} & 0 & \hat{c}_{46}^{\text {Tric }} \\
\hat{c}_{15}^{\text {Tric }} & \hat{c}_{25}^{\text {Tric }} & \hat{c}_{35}^{\text {Tric }} & 0 & \hat{c}_{55}^{\text {Tric }} & 0 \\
0 & 0 & 0 & \hat{c}_{46}^{\text {Tric }} & 0 & \hat{c}_{66}^{\text {Tric }}
\end{array}\right]
$$

For this symmetry type, equation (2.4) takes the following forms

$$
\left(\hat{C}^{V, M o n}-\lambda I\right) \hat{N}=0
$$

$$
\left[\begin{array}{cccccc}
\hat{c}_{11}^{V, \text { Mon }}-\lambda & \hat{c}_{12}^{V, \text { Mon }} & \hat{c}_{13}^{V, \text { Mon }} & 0 & \hat{c}_{15}^{V, \text { Mon }} & 0 \\
\hat{c}_{12}^{V, \text { Mon }} & \hat{c}_{22}^{V, \text { Mon }}-\lambda & \hat{c}_{23}^{V, \text { Mon }} & 0 & \hat{c}_{25}^{V, \text { Mon }} & 0 \\
\hat{c}_{13}^{V, \text { Mon }} & \hat{c}_{23}^{V, \text { Mon }} & \hat{c}_{33}^{V, \text { Mon }}-\lambda & 0 & \hat{c}_{35}^{V, \text { Mon }} & 0 \\
0 & 0 & 0 & \hat{c}_{44}^{V, \text { Mon }}-\lambda & 0 & \hat{c}_{46}^{V, \text { Mon }} \\
\hat{c}_{15}^{V, \text { Mon }} & \hat{c}_{25}^{V, \text { Mon }} & \hat{c}_{35}^{V, \text { Mon }} & 0 & \hat{c}_{55}^{V, \text { Mon }}-\lambda & 0 \\
0 & 0 & 0 & \hat{c}_{46}^{V, \text { Mon }} & 0 & \hat{c}_{66}^{V, \text { Mon }}-\lambda
\end{array}\right]\left[\begin{array}{c}
\hat{N}_{1} \\
\hat{N}_{2} \\
\hat{N}_{3} \\
\hat{N}_{4} \\
\hat{N}_{5} \\
\hat{N}_{6}
\end{array}\right]=0
$$

The Voigt eigenvalues can be obtained from the above matrix. The elements ( $\hat{s}^{R, \text { Mon }}$ ) of matrix can be expressed in terms of the elements ( $\hat{s}^{\text {Tric }}$, elastic compliances) of the triclinic symmetry by the following matrix:

$$
\hat{s}^{R, \text { Mon }}=\left[\begin{array}{cccccc}
\hat{s}_{11}^{\text {Tric }} & \hat{s}_{12}^{\text {Tric }} & \hat{s}_{13}^{\text {Tric }} & 0 & \hat{s}_{15}^{\text {Tric }} & 0 \\
\hat{s}_{12}^{\text {Tric }} & \hat{s}_{22}^{\text {Tric }} & \hat{s}_{23}^{\text {Tric }} & 0 & \hat{s}_{25}^{\text {Tric }} & 0 \\
\hat{s}_{13}^{\text {Tric }} & \hat{s}_{23}^{\text {Tric }} & \hat{s}_{33}^{\text {Tric }} & 0 & \hat{s}_{35}^{\text {Tric }} & 0 \\
0 & 0 & 0 & \hat{s}_{44}^{\text {Tric }} & 0 & \hat{s}_{46}^{\text {Tric }} \\
\hat{s}_{15}^{\text {Tric }} & \hat{s}_{25}^{\text {Tric }} & \hat{s}_{35}^{\text {Tric }} & 0 & \hat{s}_{55}^{\text {Tric }} & 0 \\
0 & 0 & 0 & \hat{s}_{46}^{\text {Tric }} & 0 & \hat{s}_{66}^{\text {Tric }}
\end{array}\right]
$$

For this symmetry type, equation (2.5) takes the following forms

$$
\left(\hat{s}^{R, \text { Mon }}-\left(\frac{1}{\lambda}\right) I\right) \hat{N}=0
$$

$$
\left[\begin{array}{cccccc}
\hat{s}_{11}^{R, \text { Mon }}-\frac{1}{\lambda} & \hat{s}_{12}^{R, \text { Mon }} & \hat{s}_{13}^{R, \text { Mon }} & 0 & \hat{s}_{15}^{R, \text { Mon }} & 0 \\
\hat{s}_{12}^{R, \text { Mon }} & \hat{s}_{22}^{R, \text { Mon }}-\frac{1}{\lambda} & \hat{s}_{23}^{R, \text { Mon }} & 0 & \hat{s}_{25}^{R, \text { Mon }} & 0 \\
\hat{s}_{13}^{R, \text { Mon }} & \hat{s}_{23}^{R, \text { Mon }} & \hat{s}_{33}^{R, \text { Mon }}-\frac{1}{\lambda} & 0 & \hat{s}_{35}^{R, \text { Mon }} & 0 \\
0 & 0 & 0 & \hat{s}_{44}^{R, \text { Mon }}-\frac{1}{\lambda} & 0 & \hat{s}_{46}^{R, \text { Mon }} \\
\hat{s}_{15}^{R, \text { Mon }} & \hat{s}_{25}^{R, \text { Mon }} & \hat{s}_{35}^{R, \text { Mon }} & 0 & \hat{s}_{55}^{R, \text { Mon }}-\frac{1}{\lambda} & 0 \\
0 & 0 & 0 & \hat{s}_{46}^{R, \text { Mon }} & 0 & \hat{s}_{66}^{R, \text { Mon }}-\frac{1}{\lambda}
\end{array}\right]\left[\begin{array}{c}
\hat{N}_{1} \\
\hat{N}_{2} \\
\hat{N}_{3} \\
\hat{N}_{4} \\
\hat{N}_{5} \\
\hat{N}_{6}
\end{array}\right]=0
$$


The Reuss eigenvalues can be obtained from above matrix.

The generalized Hill inequalities for monoclinic symmetry are

$$
\begin{array}{ll}
\lambda_{1}^{R, \text { Mon }} \leq \lambda_{1}^{e f f, \text { Mon }} \leq \lambda_{1}^{V, \text { Mon }}, & \lambda_{2}^{R, \text { Mon }} \leq \lambda_{2}^{\text {eff,Mon }} \leq \lambda_{2}^{V, \text { Mon }}, \quad \lambda_{3}^{R, \text { Mon }} \leq \lambda_{3}^{e f f, \text { Mon }} \leq \lambda_{3}^{V, \text { Mon },}, \\
\lambda_{4}^{R, \text { Mon }} \leq \lambda_{4}^{e f f, \text { Mon }} \leq \lambda_{4}^{V, \text { Mon }}, \quad \lambda_{5}^{R, \text { Mon }} \leq \lambda_{5}^{e f f, \text { Mon }} \leq \lambda_{5}^{V, \text { Mon }}, \quad \lambda_{6}^{R, \text { Mon }} \leq \lambda_{6}^{e f f, \text { Mon }} \leq \lambda_{6}^{V, \text { Mon } .}
\end{array}
$$

\subsection{For Triclinic Media}

Triclinic media show triclinic symmetry and this symmetry type is the lowest symmetry among the other anisotropic elastic symmetries. There are six distinct eigenvalues with multiplicity of one in triclinic symmetry. The elements ( $\hat{C}^{V \text {, Tric }}$ ) of matrix can be expressed in terms of the elements ( $\hat{c}^{\text {Tric }}$, elastic stiffnesses) of the triclinic symmetry by the following matrix:

$$
\hat{c}^{V, \text { Tric }}=\left[\begin{array}{llllll}
\hat{c}_{11}^{\text {Tric }} & \hat{c}_{12}^{\text {Tric }} & \hat{c}_{13}^{\text {Tric }} & \hat{c}_{14}^{\text {Tric }} & \hat{c}_{15}^{\text {Tric }} & \hat{c}_{16}^{\text {Tric }} \\
\hat{c}_{12}^{\text {Tric }} & \hat{c}_{22}^{\text {Tric }} & \hat{c}_{23}^{\text {Tric }} & \hat{c}_{24}^{\text {Tric }} & \hat{c}_{25}^{\text {Tric }} & \hat{c}_{26}^{\text {Tric }} \\
\hat{c}_{13}^{\text {Tric }} & \hat{c}_{23}^{\text {Tric }} & \hat{c}_{33}^{\text {Tric }} & \hat{c}_{34}^{\text {Tric }} & \hat{c}_{35}^{\text {Tric }} & \hat{c}_{36}^{\text {Tric }} \\
\hat{c}_{14}^{\text {Tric }} & \hat{c}_{24}^{\text {Tric }} & \hat{c}_{34}^{\text {Tric }} & \hat{c}_{44}^{\text {Tric }} & \hat{c}_{45}^{\text {Tric }} & \hat{c}_{46}^{\text {Tric }} \\
\hat{c}_{15}^{\text {Tric }} & \hat{c}_{25}^{\text {Tric }} & \hat{c}_{35}^{\text {Tric }} & \hat{c}_{45}^{\text {Tric }} & \hat{c}_{55}^{\text {Tric }} & \hat{c}_{56}^{\text {Tric }} \\
\hat{c}_{16}^{\text {Tric }} & \hat{c}_{26}^{\text {Tric }} & \hat{c}_{36}^{\text {Tric }} & \hat{c}_{46}^{\text {Tric }} & \hat{c}_{56}^{\text {Tric }} & \hat{c}_{66}^{\text {Tric }}
\end{array}\right]
$$

For this symmetry type, equation (2.4) takes the following forms

$$
\left(\hat{c}^{V, T \text { Tric }}-\lambda I\right) \hat{N}=0
$$

$$
\left[\begin{array}{cccccc}
\hat{c}_{11}^{\text {Tric }}-\lambda & \hat{c}_{12}^{\text {Tric }} & \hat{c}_{13}^{\text {Tric }} & \hat{c}_{14}^{\text {Tric }} & \hat{c}_{15}^{\text {Tric }} & \hat{c}_{16}^{\text {Tric }} \\
\hat{c}_{12}^{\text {Tric }} & \hat{c}_{22}^{\text {Tric }}-\lambda & \hat{c}_{23}^{\text {Tric }} & \hat{c}_{24}^{\text {Tric }} & \hat{c}_{25}^{\text {Tric }} & \hat{c}_{26}^{\text {Tric }} \\
\hat{c}_{13}^{\text {Tric }} & \hat{c}_{23}^{\text {Tric }} & \hat{c}_{33}^{\text {Tric }}-\lambda & \hat{c}_{34}^{\text {Tric }} & \hat{c}_{35}^{\text {Tric }} & \hat{c}_{36}^{\text {Tric }} \\
\hat{c}_{14}^{\text {Tric }} & \hat{c}_{24}^{\text {Tric }} & \hat{c}_{34}^{\text {Tric }} & \hat{c}_{44}^{\text {Tric }}-\lambda & \hat{c}_{45}^{\text {Tric }} & \hat{c}_{46}^{\text {Tric }} \\
\hat{c}_{15}^{\text {Tric }} & \hat{c}_{25}^{\text {Tric }} & \hat{c}_{35}^{\text {Tric }} & \hat{c}_{45}^{\text {Tric }} & \hat{c}_{55}^{\text {Tric }}-\lambda & \hat{c}_{56}^{\text {Tric }} \\
\hat{c}_{16}^{\text {Tric }} & \hat{c}_{26}^{\text {Tric }} & \hat{c}_{36}^{\text {Tric }} & \hat{c}_{46}^{\text {Tric }} & \hat{c}_{56}^{\text {Tric }} & \hat{c}_{66}^{\text {Tric }}-\lambda
\end{array}\right]\left[\begin{array}{c}
\hat{N}_{1} \\
\hat{N}_{2} \\
\hat{N}_{3} \\
\hat{N}_{4} \\
\hat{N}_{5} \\
\hat{N}_{6}
\end{array}\right]=0
$$

Voigt eigenvalues can be obtained from the above matrix. The elements ( $\left.\hat{S}^{R \text {,Tric }}\right)$ of matrix can be expressed in terms of the elements ( $\hat{s}^{\text {Tric }}$, elastic compliances) of the triclinic symmetry by the following matrix:

$$
\hat{s}^{R, \text { Tric }}=\left[\begin{array}{llllll}
\hat{s}_{11}^{\text {Tric }} & \hat{s}_{12}^{\text {Tric }} & \hat{s}_{13}^{\text {Tric }} & \hat{s}_{14}^{\text {Tric }} & \hat{s}_{15}^{\text {Tric }} & \hat{s}_{16}^{\text {Tric }} \\
\hat{s}_{12}^{\text {Tric }} & \hat{s}_{22}^{\text {Tric }} & \hat{s}_{23}^{\text {Tric }} & \hat{s}_{24}^{\text {Tric }} & \hat{s}_{25}^{\text {Tric }} & \hat{s}_{26}^{\text {Tric }} \\
\hat{s}_{13}^{\text {Tric }} & \hat{s}_{23}^{\text {Tric }} & \hat{s}_{33}^{\text {Tric }} & \hat{s}_{34}^{\text {Tric }} & \hat{s}_{35}^{\text {Tric }} & \hat{s}_{36}^{\text {Tric }} \\
\hat{s}_{14}^{\text {Tric }} & \hat{s}_{24}^{\text {Tric }} & \hat{s}_{34}^{\text {Tric }} & \hat{s}_{44}^{\text {Tric }} & \hat{s}_{45}^{\text {Tric }} & \hat{s}_{46}^{\text {Tric }} \\
\hat{s}_{15}^{\text {Tric }} & \hat{s}_{25}^{\text {Tric }} & \hat{s}_{35}^{\text {Tric }} & \hat{s}_{45}^{\text {Tric }} & \hat{s}_{55}^{\text {Tric }} & \hat{s}_{56}^{\text {Tric }} \\
\hat{s}_{16}^{\text {Tric }} & \hat{s}_{26}^{\text {Tric }} & \hat{s}_{36}^{\text {Tric }} & \hat{s}_{46}^{\text {Tric }} & \hat{s}_{56}^{\text {Tric }} & \hat{s}_{66}^{\text {Tric }}
\end{array}\right]
$$

For this symmetry type, equation (2.5) takes the following forms 
$\left(\hat{s}^{R, T r i C}-\left(\frac{1}{\lambda}\right) I\right) \hat{N}=0$

$\left[\begin{array}{cccccc}\hat{s}_{11}^{\text {Tric }}-\frac{1}{\lambda} & \hat{s}_{12}^{\text {Tric }} & \hat{s}_{13}^{\text {Tric }} & \hat{s}_{14}^{\text {Tric }} & \hat{s}_{15}^{\text {Tric }} & \hat{s}_{16}^{\text {Tric }} \\ \hat{s}_{12}^{\text {Tric }} & \hat{s}_{22}^{\text {Tric }}-\frac{1}{\lambda} & \hat{s}_{23}^{\text {Tric }} & \hat{s}_{24}^{\text {Tric }} & \hat{s}_{25}^{\text {Tric }} & \hat{s}_{26}^{\text {Tric }} \\ \hat{s}_{13}^{\text {Tric }} & \hat{s}_{23}^{\text {Tric }} & \hat{s}_{33}^{\text {Tric }}-\frac{1}{\lambda} & \hat{s}_{34}^{\text {Tric }} & \hat{s}_{35}^{\text {Tric }} & \hat{s}_{36}^{\text {Tric }} \\ \hat{s}_{14}^{\text {Tric }} & \hat{s}_{24}^{\text {Tric }} & \hat{s}_{34}^{\text {Tric }} & \hat{s}_{44}^{\text {Tric }}-\frac{1}{\lambda} & \hat{s}_{45}^{\text {Tric }} & \hat{s}_{46}^{\text {Tric }} \\ \hat{s}_{15}^{\text {Tric }} & \hat{s}_{25}^{\text {Tric }} & \hat{s}_{35}^{\text {Tric }} & \hat{s}_{45}^{\text {Tric }} & \hat{s}_{55}^{\text {Tric }}-\frac{1}{\lambda} & \hat{s}_{56}^{\text {Tric }} \\ \hat{s}_{16}^{\text {Tric }} & \hat{s}_{26}^{\text {Tric }} & \hat{s}_{36}^{\text {Tric }} & \hat{s}_{46}^{\text {Tric }} & \hat{s}_{56}^{\text {Tric }} & \hat{s}_{66}^{\text {Tric }}-\frac{1}{\lambda}\end{array}\right]\left[\begin{array}{l}\hat{N}_{1} \\ \hat{N}_{2} \\ \hat{N}_{3} \\ \hat{N}_{4} \\ \hat{N}_{5} \\ \hat{N}_{6}\end{array}\right]=0$

Reuss eigenvalues can be obtained from the above matrix.

The generalized Hill inequalities for triclinic symmetry are

$\lambda_{1}^{R \text {,Tric }} \leq \lambda_{1}^{\text {eff, } \text {,Tric }} \leq \lambda_{1}^{V \text {,Tric }}, \quad \lambda_{2}^{R \text {,Tric }} \leq \lambda_{2}^{\text {eff, Tric }} \leq \lambda_{2}^{V, \text { Tric }}, \quad \lambda_{3}^{R, \text { Tric }} \leq \lambda_{3}^{\text {eff, Tric }} \leq \lambda_{3}^{V \text {,Tric }}, \quad \lambda_{4}^{R \text {,Tric }} \leq \lambda_{4}^{\text {eff ,Tric }} \leq \lambda_{4}^{V \text {,Tric }}$, $\lambda_{5}^{R, \text { Tric }} \leq \lambda_{5}^{\text {eff, Tric }} \leq \lambda_{5}^{V \text {,Tric }}, \quad \lambda_{6}^{R, \text { Tric }} \leq \lambda_{6}^{\text {eff, Tric }} \leq \lambda_{6}^{V, \text { Tric }}$.

\section{Numerical Examples for Various Types of Anisotropic Elastic Symmetries}

The elastic constants of randomly selected materials are given for each corresponding anisotropic symmetry type (taken from Landolt-Bö rnstein (1979)). These data are used to calculate the lower and upper bounds on effective eigenvalues for all types of materials. For all anisotropic symmetry types, the units of Voigt elastic constant data, eigenvalues are GPa $=10^{10} \mathrm{dyn} \cdot \mathrm{cm}^{-2}$ and the units of Reuss elastic constant data, eigenvalues are

$(\mathrm{TPa})^{-1}=10^{-13} \mathrm{~cm}^{2} \cdot \mathrm{dyn}^{-1}$.

\subsection{For Cubic Media}

Table 5.1: Voigt elastic constant data of cubic media

\begin{tabular}{|c|c|c|c|}
\hline Cubic Media & $\hat{\mathbf{c}}_{11}^{V, \text { Cub }}$ & $\hat{\mathbf{c}}_{12}^{V, C u b}$ & $\hat{\mathbf{c}}_{44}^{\text {, Cub }}$ \\
\hline Diamond,C (Grimsditch et al.(1975)) & 1040 & 170 & 550 \\
\hline Platinum(Pt) (Macfarlane et al.(1965)) & 347 & 251 & 76.5 \\
\hline Beryllium oxide (BeO) (Martin (1972)) & 381 & 147 & 200 \\
\hline $\begin{array}{c}\text { Rubidium silver iodide(RbAg } \mathbf{I}_{5} \text { ) (Graham and Chang } \\
(1975) \text { ) }\end{array}$ & 16.5 & 9.34 & 4.89 \\
\hline $\begin{array}{c}\text { Thallium manganese chloride(TIMnCl } \\
\text { (1975)) }\end{array}$ & 44.8 & 28.3 & 16.1 \\
\hline
\end{tabular}

Table 5.2: Reuss elastic constant data of cubic media

\begin{tabular}{|c|c|c|c|}
\hline Cubic Media & $\hat{\mathbf{s}}_{11}^{R, C u b}$ & $\hat{\mathbf{s}}_{12}^{R, C u b}$ & $\hat{\mathbf{s}}_{44}^{R, C u b}$ \\
\hline Diamond,C (Grimsditch et al.(1975)) & 1.01 & -0.14 & 1.83 \\
\hline Platinum(Pt) (Macfarlane et al.(1965)) & & & \\
& 7.35 & 23.08 & 13.1 \\
\hline Beryllium oxide(BeO) (Martin (1972)) & 3.35 & -0.93 & 5.01 \\
\hline
\end{tabular}




\begin{tabular}{|c|c|c|c|}
\hline $\begin{array}{l}\text { Rubidium silver iodide( } \mathbf{R b} \mathbf{A g}_{4} \mathbf{I}_{5} \text { ) (Graham and Chang } \\
\text { (1975)) }\end{array}$ & 103 & -37 & 204 \\
\hline $\begin{array}{l}\text { Thallium manganese chloride(TIMnCl }{ }_{3} \text { ) (Aleksandrov } \\
\text { (1975)) }\end{array}$ & 43.7 & -16.9 & 62.1 \\
\hline
\end{tabular}

Table 5.3: Lower bounds on effective eigenvalues of cubic media

\begin{tabular}{|c|c|c|c|}
\hline Cubic Media & $\lambda_{1}^{R, C u b}$ & $\lambda_{(2,3)}^{R, C u b}$ & $\lambda_{(4,5,6)}^{R, C u b}$ \\
\hline Diamond,C & 1369.9 & 896.6 & 1092.9 \\
\hline Platinum(Pt) & 840.3 & 95.9 & 152.7 \\
\hline Beryllium oxide(BeO) & 671.1 & 233.6 & 399.2 \\
\hline Rubidium silver iodide( $\mathrm{RbAg}_{4} \mathbf{I}_{5}$ ) & 34.5 & 7.10 & 9.80 \\
\hline Thallium manganese chloride( $\mathrm{TIMnCl}_{3}$ ) & 101 & 16.5 & 32.2 \\
\hline
\end{tabular}

Table 5.4: Upper bounds on effective eigenvalues of cubic media

\begin{tabular}{|c|c|c|c|}
\hline Cubic Media & $\lambda_{1}^{V, \mathrm{Cub}}$ & $\lambda_{(2,3)}^{V, \mathrm{Cub}}$ & $\lambda_{(4,5,6)}^{V, \mathrm{Cub}}$ \\
\hline Diamond,C & 1380 & 870 & 1100 \\
\hline Platinum(Pt) & 849 & 96 & 153 \\
\hline Beryllium oxide(BeO) & 675 & 234 & 400 \\
\hline${\text { Rubidium silver iodide(RbAg }{ }_{4} \mathbf{I}_{{ }_{5}} \mathbf{~}}_{\text {Thallium manganese chloride(TIMnCI }_{{ }_{3}} \mathbf{T}}$ & 35.2 & 7.16 & 9.80 \\
\hline
\end{tabular}

\subsection{For Isotropic Media}

For some materials, it is possible to make approaches from cubic symmetry to isotropic symmetry. With cubic symmetry, three independent elastic constants are needed. If the medium is elastically isotropic, the elastic properties are independent of direction

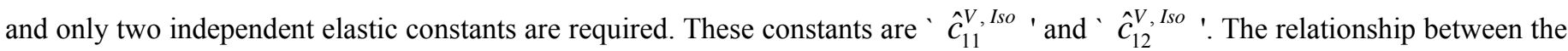
cubic and isotropic symmetry is $2 * \hat{c}_{44}^{V \text {, Cub }}=\hat{c}_{11}^{V \text {, Cub }}-\hat{c}_{12}^{V \text {, Cub }}$ '. By this equality, we can get the anisotropy ratio which is demostrated by $\mathrm{A}$ and $A=\frac{2 \cdot \hat{c}_{44}^{V, C u b}}{\left(\hat{c}_{11}^{V, C u b}-\hat{c}_{12}^{V, C u b}\right)} \cdot A$ is unitless. The degree of anisotropy is measured by the deviation of $A$ from the value $A=1$, characteristic of an isotropic medium. If the deviation from 1 is small, then we can say that the material is practically isotropic. Voigt and Reuss elastic constants of some nearly isotropic materials are given in Tables 5.5 and 5.6 respectively.

Table 5.5: Voigt elastic constant data of isotropic media

\begin{tabular}{|c|c|c|c|c|}
\hline Isotropic Media & $\hat{\mathbf{c}}_{11}^{V, \text { Cub }}$ & $\hat{\mathbf{c}}_{12}^{V, \text { Cub }}$ & $\hat{\mathbf{c}}_{44}^{V, \text { Cub }}$ & A \\
\hline Aluminium (Landolt-Bornstein (1979)) & 108 & 62 & 28.3 & 1.23 \\
\hline $\begin{array}{c}\text { Alloy: Aluminium-magnesium at \%7.7 Mg (Gault et } \\
\text { al.(1977)) }\end{array}$ & 103 & 57 & 29 & 1.26 \\
\hline $\begin{array}{c}\text { Alloy: Aluminium-magnesium at \%4.5 Mg (Gault et } \\
\text { al.(1977)) }\end{array}$ & 104 & 58 & 28.8 & 1.25 \\
\hline Alloy:Titanium-Vanadium at \%53 V (Fisher (1975)) & 177.3 & 114.7 & 41.3 & 1.32 \\
\hline Alloy:Lead-indium at \%9 In (Van Der Planken (1971)) & 59.70 & 33.70 & 13.90 & 1.10 \\
\hline
\end{tabular}

Table 5.6: Reuss elastic constant data of isotropic media

\begin{tabular}{|c|c|c|c|}
\hline Isotropic Media & $\hat{\mathbf{s}}_{11}^{R, C u b}$ & $\hat{\mathbf{s}}_{12}^{R, C u b}$ & $\hat{\mathbf{s}}_{44}^{R, C u b}$ \\
\hline Aluminium (Landolt-Bornstein (1979)) & 16 & -5.8 & 35.3 \\
\hline $\begin{array}{c}\text { Alloy: Aluminium-magnesium at \%7.7 Mg (Gault et } \\
\text { al.(1977)) }\end{array}$ & 16 & -5.7 & 34.5 \\
\hline
\end{tabular}




\begin{tabular}{|c|c|c|c|}
\hline $\begin{array}{c}\text { Alloy: Aluminium-magnesium at \%4.5 Mg (Gault et } \\
\text { al.(1977)) }\end{array}$ & 16 & -5.7 & 34.7 \\
\hline Alloy:Titanium-Vanadium at \%53 V (Fisher (1975)) & 11.5 & -4.5 & 24.2 \\
\hline Alloy:Lead-indium at \%9 In (Van Der Planken (1971)) & 28.3 & -10.2 & 71.9 \\
\hline
\end{tabular}

Table 5.7: Lower bounds on effective eigenvalues of isotropic media

\begin{tabular}{|c|c|c|c|}
\hline Isotropic Media & $\lambda_{1}^{R, C u b}$ & $\lambda_{(2,3)}^{R, C u b}$ & $\lambda_{(4,5,6)}^{R, C u b}$ \\
\hline Aluminium & 227 & 45.9 & 56.8 \\
\hline Alloy: Aluminium-magnesium at \%7.7 Mg & 217 & 46 & 58 \\
\hline Alloy: Aluminium-magnesium at \%4.5 Mg & 217.4 & 46 & 57.6 \\
\hline Alloy:Titanium-Vanadium at \%53 V & 400 & 62.5 & 82.6 \\
\hline Alloy:Lead-indium at \%9 In & 126.6 & 26 & 27.8 \\
\hline
\end{tabular}

Table 5.8: Upper bounds on effective eigenvalues of isotropic media

\begin{tabular}{|l|l|l|l|}
\hline Isotropic Media & $\lambda_{1}^{V, C u b}$ & $\lambda_{(2,3)}^{V, C u b}$ & $\lambda_{(4,5,6)}^{V, C u b}$ \\
\hline Aluminium & 232 & 46 & 56.8 \\
\hline Alloy: Aluminium-magnesium at \%7.7 Mg & 217 & 46 & 58 \\
\hline Alloy: Aluminium-magnesium at \%4.5 Mg & 220 & 46 & 57.6 \\
\hline Alloy:Titanium-Vanadium at \%53 V & 406.7 & 62.6 & 82.6 \\
\hline Alloy:Lead-indium at \%9 In & 127.1 & 26 & 27.8 \\
\hline
\end{tabular}

\subsection{For Tetragonal Media}

Table 5.9: Voigt elastic constant data of tetragonal media

\begin{tabular}{|c|c|c|c|c|c|c|}
\hline Tetragonal Media & $\hat{\mathbf{c}}_{11}^{V, T e t}$ & $\hat{\mathbf{c}}_{12}^{V, T e t}$ & $\hat{\mathbf{c}}_{13}^{V, T e t}$ & $\hat{\mathbf{c}}_{33}^{V, T e t}$ & $\hat{\mathbf{C}}_{44}^{V, T e t}$ & $\hat{\mathbf{c}}_{66}^{V, T e t}$ \\
\hline $\begin{array}{l}\text { Indium-cadmium alloy, In-3.42 at \%Cd (Madhava anc } \\
\text { Saunders (1977)) }\end{array}$ & 44.8 & 41 & 40.5 & 44.1 & 6.86 & 11.25 \\
\hline $\begin{array}{l}\text { Ammonium dihydrogen arsenate (piezoel.), } \mathbf{N H}_{4} \mathbf{H}_{2} \\
\text { ASO }_{4} \text { (Haussü } \mathrm{hl} \text { (1964)) }\end{array}$ & 62.2 & 8.6 & 18.4 & 29.6 & 6.69 & 6.22 \\
\hline 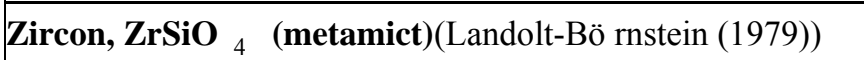 & 284 & 73 & 119 & 309 & 77.5 & 47.7 \\
\hline
\end{tabular}

Table 5.10: Reuss elastic constant data of tetragonal media

\begin{tabular}{|l|l|l|l|l|l|l|}
\hline Tetragonal Media & $\hat{\mathbf{s}}_{11}^{R, T e t}$ & $\hat{\mathbf{s}}_{12}^{R, T e t}$ & $\hat{\mathbf{s}}_{13}^{R, T e t}$ & $\hat{\mathbf{s}}_{33}^{R, T e t}$ & $\hat{\mathbf{s}}_{44}^{R, T e t}$ & $\hat{\mathbf{s}}_{66}^{R, T e t}$ \\
\hline $\begin{array}{l}\text { Indium-cadmium alloy, In-3.42 at \%Cd (Madhava and } 174 \\
\text { Saunders (1977)) }\end{array}$ & -86 & -81 & 172 & 146 & 89 \\
\hline $\begin{array}{l}\text { Ammonium dihydrogen arsenate (piezoel.), NH }{ }_{4} \mathbf{H}_{2} \\
\text { ASO }\end{array}$ (Haussü hl (1964)) $^{\text {Zircon, ZrSiO }}{ }_{4}$ (metamict)(Landolt-Bö rnstein (1979)) & 4.26 & 1.1 & -13 & 50 & 149 & 161 \\
\hline
\end{tabular}

Table 5.11:Lower bounds on effective eigenvalues of tetragonal media

\begin{tabular}{|c|c|c|c|c|c|}
\hline Tetragonal Media & $\lambda_{1}^{R, T e t}$ & $\lambda_{2}^{R, \text { Tet }}$ & $\lambda_{3}^{R, T e t}$ & $\lambda_{(4,5)}^{R, T e t}$ & $\lambda_{6}^{R, T e t}$ \\
\hline Indium-cadmium alloy, In-3.42 at \%Cd & 125.1 & 4 & 3.80 & 13.7 & 22.5 \\
\hline $\begin{array}{l}{\text { Ammonium dihydrogen arsenate (piezoel.), } \mathrm{NH}_{4}} \mathrm{H}_{2} \\
\text { ASO }_{4}\end{array}$ & 83.3 & 17 & 53.5 & 13.4 & 12.4 \\
\hline 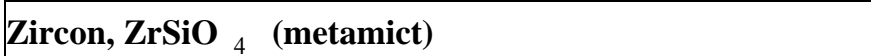 & 501.7 & 163 & 210.5 & 155 & 95.2 \\
\hline
\end{tabular}


Table 5.12: Upper bounds on effective eigenvalues of tetragonal media

\begin{tabular}{|c|c|c|c|c|c|}
\hline Tetragonal Media & $\lambda_{1}^{V, T e t}$ & $\lambda_{2}^{V, T e t}$ & $\lambda_{3}^{V, T e t}$ & $\lambda_{(4,5)}^{V, T e t}$ & $\lambda_{6}^{V, T e t}$ \\
\hline Indium-cadmium alloy, In-3.42 at \%Cd & 125.3 & 4 & 3.80 & 13.7 & 22.5 \\
\hline $\begin{array}{l}{\text { Ammonium dihydrogen arsenate (piezoel.), } \mathrm{NH}_{4}}_{{ }_{4}} \mathrm{H}_{2} \\
\text { ASO }_{4}\end{array}$ & 83.4 & 17 & 53.6 & 13.4 & 12.4 \\
\hline 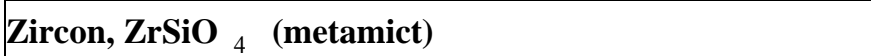 & 502.9 & 163 & 211 & 155 & 95.4 \\
\hline
\end{tabular}

\subsection{For Transversely Isotropic Media}

Table 5.13:Voigt elastic constant data of transversely isotropic media

\begin{tabular}{|l|l|l|l|l|l|}
\hline Transversely Isotropic Media & $\hat{\mathbf{c}}_{11}^{V, \text { Trans }}$ & $\hat{\mathbf{c}}_{12}^{V, \text { Trans }}$ & $\hat{\mathbf{c}}_{13}^{V, \text { Trans }}$ & $\hat{\mathbf{c}}_{33}^{V, \text { Trans }}$ & $\hat{\mathbf{c}}_{44}^{V, \text { Trans }}$ \\
\hline Cobalt(Co)(Masumoto et al. (1967)) & 295 & 159 & 111 & 335 & 71 \\
\hline Hafnium(Hf) (Fisher and Renken (1964)) & 181 & 77 & 66 & 197 & 55.7 \\
\hline Zinc(Zn)(Singh et al.(1977)) & 165 & 31.1 & 50 & 61.8 & 39.6 \\
\hline Bone(dried phalanx)(Bonfield and Grynpas (1977)) & 21.2 & 9.50 & 10.2 & 37.4 & 7.50 \\
\hline Polystyrene(Wright et al. (1971)) & 5.20 & 2.75 & 2.75 & 5.70 & 1.30 \\
\hline
\end{tabular}

Table 5.14:Reuss elastic constant data of transversely isotropic media

\begin{tabular}{|l|l|l|l|l|l|}
\hline Transversely Isotropic Media & $\hat{\mathbf{s}}_{11}^{R, \text { Trans }}$ & $\hat{\mathbf{s}}_{12}^{R, \text { Trans }}$ & $\hat{\mathbf{s}}_{13}^{R, \text { Trans }}$ & $\hat{\mathbf{s}}_{33}^{R, \text { Trans }}$ & $\hat{\mathbf{s}}_{44}^{R, \text { Trans }}$ \\
\hline Cobalt(Co)(Masumoto et al. (1967)) & 5.11 & -2.37 & -0.94 & 3.69 & 14.1 \\
\hline Hafnium(Hf) (Fisher and Renken (1964)) & 7.16 & -2.48 & -1.57 & 6.13 & 18 \\
\hline Zinc(Zn)(Singh et al.(1977)) & 8.22 & 0.60 & -7 & 27.7 & 25.3 \\
\hline Bone(dried phalanx)(Bonfield and Grynpas (1977)) & 63 & -23 & -11 & 33 & 133 \\
\hline Polystyrene(Wright et al. (1971)) & 299 & -109 & -91 & 264 & 770 \\
\hline
\end{tabular}

Table 5.15:Lower bounds on effective eigenvalues of transversely isotropic media

\begin{tabular}{|l|l|l|l|l|}
\hline Transversely Isotropic Media & $\lambda_{1}^{R, \text { Trans }}$ & $\lambda_{2}^{R, \text { Trans }}$ & $\lambda_{(3,6)}^{R, \text { Trans }}$ & $\lambda_{(4,5)}^{R, \text { Trans }}$ \\
\hline Cobalt(Co) & 554.5 & 216 & 133.7 & 141.8 \\
\hline Hafnium(H) & 324.2 & 129.3 & 103.3 & 111.1 \\
\hline Zinc(Zn) & 218.3 & 31.3 & 131.2 & 79.1 \\
\hline Bone(dried phalanx) & 48.7 & 19.1 & 11.6 & 15 \\
\hline Polystyrene & 10.7 & 2.77 & 2.45 & 2.60 \\
\hline
\end{tabular}

Table 5.16:Upper bounds on effective eigenvalues of transversely isotropic media

\begin{tabular}{|l|l|l|l|l|}
\hline Transversely Isotropic Media & $\lambda_{1}^{V, \text { Trans }}$ & $\lambda_{2}^{V, \text { Trans }}$ & $\lambda_{(3,6)}^{V, \text { Trans }}$ & $\lambda_{(4,5)}^{V, \text { Trans }}$ \\
\hline Cobalt(Co) & 562.4 & 226.6 & 136 & 142 \\
\hline Hafnium(Hf) & 325.7 & 129.3 & 104 & 111.4 \\
\hline Zinc(Zn) & 226.5 & 31.4 & 133.9 & 79.2 \\
\hline Bone(dried phalanx) & 48.9 & 19.2 & 11.7 & 15 \\
\hline Polystyrene & 10.9 & 2.78 & 2.45 & 2.6 \\
\hline
\end{tabular}

\subsection{For Trigonal Media}

Table 5.17: Voigt elastic constant data of trigonal media

\begin{tabular}{|l|l|l|l|l|l|l|}
\hline Trigonal Media & $\hat{\mathbf{c}}_{11}^{V, \text { Trig }}$ & $\hat{\mathbf{c}}_{12}^{V, \text { Trig }}$ & $\hat{\mathbf{c}}_{13}^{V, \text { Trig }}$ & $\hat{\mathbf{c}}_{14}^{V, \text { Trig }}$ & $\hat{\mathbf{c}}_{33}^{V, \text { Trig }}$ & $\hat{\mathbf{c}}_{44}^{V, \text { Trig }}$ \\
\hline Antimony(Epstein (1965)) & 99.4 & 30.9 & 26.4 & 21.6 & 44.5 & 39.5 \\
\hline Magnesite,MgCO ${ }_{3}$ (Humbert(1972)) & 259 & 75.6 & 58.8 & -19 & 156 & 54.8 \\
\hline Haematite,Fe ${ }_{2} \mathbf{O}_{3}$ (Landolt-Bö rnstein(1979)) & 242 & 54.9 & 15.7 & -12.7 & 228 & 85.3 \\
\hline As-Sb at \% As 25.5(Akgö z et al.(1976)) & 106.7 & 48.4 & 28.5 & 18.8 & 48 & 40.8 \\
\hline Arsenic(Pace and Saunders (1971)) & 130.2 & 30.3 & 64.3 & -3.71 & 58.7 & 22.5 \\
\hline
\end{tabular}


Table 5.18: Reuss elastic constant data of trigonal media

\begin{tabular}{|c|c|c|c|c|c|c|}
\hline Trigonal Media & $\hat{\mathbf{S}}_{11}^{R, \text { Trig }}$ & $\hat{\mathbf{s}}_{12}^{R, \text { Trig }}$ & $\hat{\mathbf{s}}_{13}^{R, \text { Trig }}$ & $\hat{\mathbf{S}}_{14}^{R, \text { Trig }}$ & $\hat{\mathbf{s}}_{33}^{R, \text { Trig }}$ & $\hat{\mathbf{S}}_{44}^{R, \text { Trig }}$ \\
\hline Antimony(Epstein (1965)) & 16.2 & -6.1 & -5.9 & -12.2 & 29.5 & 38.6 \\
\hline Magnesite,MgCO ${ }_{3}($ Humbert(1972)) & 4.67 & -1.22 & -1.30 & 2.04 & 7.41 & 19.7 \\
\hline Haematite,Fe ${ }_{2} \mathbf{O}_{3}$ (Landolt-Bö rnstein(1979)) & 4.41 & -1.02 & -0.23 & 0.79 & 4.43 & 11.9 \\
\hline As-Sb at \% As 25.5(Akgö z et al.(1976)) & 15.4 & -6.96 & -4.96 & -9.76 & 27 & 33.3 \\
\hline Arsenic(Pace and Saunders (1971)) & 30.3 & 20.2 & -55.2 & 1.67 & 137.8 & 45 \\
\hline
\end{tabular}

Table 5.19: Lower bounds on effective eigenvalues of trigonal media

\begin{tabular}{|l|l|l|l|l|}
\hline Trigonal Media & $\lambda_{1}^{R, \text { Trig }}$ & $\lambda_{2}^{R, \text { Trig }}$ & $\lambda_{(3,6)}^{R, \text { Trig }}$ & $\lambda_{(4,5)}^{R, \text { Trig }}$ \\
\hline Antimony & 142.8 & 30.5 & 57.7 & 12.2 \\
\hline Magnesite,MgCO & 366.6 & 123 & 177.2 & 25.2 \\
\hline Haematite,Fe ${ }_{2} \mathbf{O}_{3}$ & 303.3 & 221.1 & 186.5 & 41.9 \\
\hline As-Sb at \% As 25.5 & 164.3 & 34.1 & 54.3 & 14.2 \\
\hline Arsenic & 212.3 & 5.4 & 99.7 & 11.1 \\
\hline
\end{tabular}

Table 5.20: Upper bounds on effective eigenvalues of trigonal media

\begin{tabular}{|l|l|l|l|l|}
\hline Trigonal Media & $\lambda_{1}^{V, \text { Trig }}$ & $\lambda_{2}^{V, \text { Trig }}$ & $\lambda_{(3,6)}^{V, \text { Trig }}$ & $\lambda_{(4,5)}^{V, \text { Trig }}$ \\
\hline Antimony & 144.3 & 30.5 & 117.3 & 30.2 \\
\hline Magnesite,MgCO ${ }_{3}$ & 367.3 & 123.3 & 199.5 & 93.5 \\
\hline Haematite,Fe ${ }_{2} \mathbf{O}_{3}$ & 303.4 & 221.5 & 205.6 & 152.1 \\
\hline As-Sb at \% As 25.5 & 168.6 & 34.5 & 109.3 & 30.6 \\
\hline Arsenic & 213.8 & 5.39 & 100.9 & 44 \\
\hline
\end{tabular}

\subsection{For Orthorhombic Media}

Table 5.21: Voigt elastic constant data of orthorhombic media

\begin{tabular}{|l|l|l|l|l|l|}
\hline Orthorhombic Media & $\hat{\mathbf{c}}_{11}^{V, \text { Ort }}$ & $\hat{\mathbf{c}}_{12}^{V, \text { Ort }}$ & $\hat{\mathbf{c}}_{13}^{V, \text { Ort }}$ & $\hat{\mathbf{c}}_{22}^{V, \text { Ort }}$ & $\hat{\mathbf{c}}_{23}^{V, \text { Ort }}$ \\
\hline Pine(Softwood)(Landolt-Bö rnstein(1979)) & 1.24 & 0.74 & 0.76 & 17.1 & 0.94 \\
\hline Olivinite(Landolt-Bö rnstein(1979)) & 232 & 93 & 92 & 210 & 82 \\
\hline Maple(Hardwood)(Kollmann (1968)) & 1.45 & 1.20 & 1.27 & 2.57 & 1.82 \\
\hline Human tibia(Cowin (1989)) & 11.4 & 7.73 & 5.99 & 14.1 & 6.74 \\
\hline Sodium nitrite(piezoel), NaNO ${ }_{2}$ (Shimizu (1974)) & 30.6 & 12.5 & 15.6 & 56.3 & 14.6 \\
\hline Rubidium sulfate, Rb ${ }_{2}$ SO $_{4}$ (Haussü hl (1965)) & 50.3 & 19.6 & 20 & 51 & 19.2 \\
\hline Human femoral cortical bone(Cowin and Sadegh (1991)) & 18 & 9.98 & 10.1 & 20.2 & 10.7 \\
\hline
\end{tabular}

\begin{tabular}{|l|l|l|l|l|}
\hline Orthorhombic Media & $\hat{\mathbf{c}}_{33}^{V, \text { Ort }}$ & $\hat{\mathbf{c}}_{44}^{V, \text { Ort }}$ & $\hat{\mathbf{c}}_{55}^{V, \text { Ort }}$ & $\hat{\mathbf{c}}_{66}^{V, \text { Ort }}$ \\
\hline Pine(Softwood) & 1.79 & 1.18 & 0.079 & 0.91 \\
\hline Olivinite & 199 & 73.3 & 70.9 & 68.6 \\
\hline Maple(Hardwood) & 11.5 & 1.23 & 1.10 & 0.292 \\
\hline Human tibia & 22.4 & 4.91 & 3.65 & 2.41 \\
\hline Sodium nitrite(piezoel) & 63.9 & 11.7 & 9.90 & 5 \\
\hline Rubidium sulfate & 47.6 & 16.3 & 15.9 & 14.1 \\
\hline Human femoral cortical bone & 27.6 & 6.23 & 5.61 & 4.52 \\
\hline
\end{tabular}


Table 5.22: Reuss elastic constant data of orthorhombic media

\begin{tabular}{|l|l|l|l|l|l|}
\hline Orthorhombic Media & $\hat{\mathbf{s}}_{11}^{R, \text { Ort }}$ & $\hat{\mathbf{s}}_{12}^{R, \text { Ort }}$ & $\hat{\mathbf{s}}_{13}^{R, \text { Ort }}$ & $\hat{\mathbf{s}}_{22}^{R, \text { Ort }}$ & $\hat{\mathbf{s}}_{23}^{R, \text { Ort }}$ \\
\hline Pine(Softwood)(Landolt-Bö rnstein(1979)) & 1110 & -23 & -464 & 61 & -22 \\
\hline Olivinite(Landolt-Bö rnstein(1979)) & 5.85 & -1.84 & -1.95 & 6.25 & -1.71 \\
\hline Maple(Hardwood)(Kollmann (1968)) & 1.15 & -0.50 & -0.05 & 0.658 & -0.049 \\
\hline Human tibia(Cowin (1989)) & 0.145 & -0.071 & -0.017 & 0.118 & -0.016 \\
\hline Sodium nitrite(piezoel), NaNO ${ }_{2}$ (Shimizu (1974)) & 39.8 & -6.7 & -8.20 & 20.1 & -3 \\
\hline Rubidium sulfate, Rb ${ }_{2}$ SO $_{4}$ (Haussü hl (1965)) & 25.8 & -6.9 & -8 & 25.1 & -7.3 \\
\hline Human femoral cortical bone(Cowin and Sadegh (1991)) & 0.0833 & -0.0315 & -0.018 & 0.0742 & -0.0173 \\
\hline
\end{tabular}

\begin{tabular}{|l|l|l|l|l|}
\hline Orthorhombic Media & $\hat{\mathbf{s}}_{33}^{R, \text { Ort }}$ & $\hat{\mathbf{s}}_{44}^{R, \text { Ort }}$ & $\hat{\mathbf{s}}_{55}^{R, \text { Ort }}$ & $\hat{\mathbf{s}}_{66}^{R, \text { Ort }}$ \\
\hline Pine(Softwood) & 770 & 850 & 12580 & 1098 \\
\hline Olivinite & 6.61 & 13.7 & 14.2 & 14.6 \\
\hline Maple(Hardwood) & 0.100 & 0.813 & 0.909 & 3.42 \\
\hline Human tibia & 0.054 & 0.204 & 0.274 & 0.415 \\
\hline Sodium nitrite(piezoel),NaNO & 18.5 & 86 & 101 & 202 \\
\hline Rubidium sulfate, Rb ${ }_{2}$ SO $_{4}$ & 27.4 & 61.5 & 62.9 & 71.1 \\
\hline Human femoral cortical bone & 0.0496 & 0.161 & 0.178 & 0.221 \\
\hline
\end{tabular}

Table 5.23: Lower bounds on effective eigenvalues of orthorhombic media

\begin{tabular}{|l|l|l|l|l|l|l|}
\hline Orthorhombic Media & $\lambda_{1}^{R, \text { Ort }}$ & $\lambda_{2}^{R, \text { Ort }}$ & $\lambda_{3}^{R, \text { Ort }}$ & $\lambda_{4}^{R, \text { Ort }}$ & $\lambda_{5}^{R, \text { Ort }}$ & $\lambda_{6}^{R, \text { Ort }}$ \\
\hline Pine(Softwood) & 17.1 & 0.697 & 2.23 & 2.40 & 0.16 & 1.82 \\
\hline Olivinite & 393 & 121 & 126.6 & 146 & 140.8 & 137 \\
\hline Maple(Hardwood) & 12.1 & 0.686 & 2.77 & 2.46 & 2.19 & 0.584 \\
\hline Human tibia & 30.5 & 4.91 & 12.4 & 9.82 & 7.30 & 4.82 \\
\hline Sodium nitrite(piezoel),NaNO $\mathbf{N a}_{2}$ & 82.4 & 22.8 & 44.6 & 23.3 & 19.8 & 9.9 \\
\hline Rubidium sulfate, $\mathbf{R b}_{2} \mathbf{S O}_{4}$ & 88.6 & 28.7 & 31.1 & 32.5 & 31.8 & 28.1 \\
\hline Human femoral cortical bone & 43.1 & 9.035 & 13.7 & 12.4 & 11.2 & 9.04 \\
\hline
\end{tabular}

Table 5.24: Upper bounds on effective eigenvalues of orthorhombic media

\begin{tabular}{|l|l|l|l|l|l|l|}
\hline Orthorhombic Media & $\lambda_{1}^{V, \text { Ort }}$ & $\lambda_{2}^{V, \text { Ort }}$ & $\lambda_{3}^{V, \text { Ort }}$ & $\lambda_{4}^{V, \text { Ort }}$ & $\lambda_{5}^{V, \text { Ort }}$ & $\lambda_{6}^{V, \text { Ort }}$ \\
\hline Pine(Softwood) & 17.2 & 0.71 & 2.23 & 2.40 & 0.16 & 1.82 \\
\hline Olivinite & 393 & 121 & 127.4 & 146.6 & 141.8 & 137.2 \\
\hline Maple(Hardwood) & 12.1 & 0.686 & 2.77 & 2.46 & 2.19 & 0.584 \\
\hline Human tibia & 30.5 & 4.91 & 12.4 & 9.82 & 7.30 & 4.82 \\
\hline Sodium nitrite(piezoel),NaNO $\mathbf{N a}_{2}$ & 82.8 & 23 & 45 & 23.4 & 19.8 & 10 \\
\hline Rubidium sulfate, $\mathbf{R b}_{2} \mathbf{S O}_{4}$ & 88.9 & 28.8 & 31.2 & 32.6 & 31.8 & 28.2 \\
\hline Human femoral cortical bone & 43.1 & 9.041 & 13.7 & 12.5 & 11.2 & 9.04 \\
\hline
\end{tabular}

\subsection{For Monoclinic Media}

\begin{tabular}{|c|c|c|}
\hline Monoclinic Media & $\begin{array}{l}\text { Coesite, } \\
(1977))\end{array}$ & $\begin{array}{l}\text { Diphenyl, } \mathbf{C}_{12} \mathbf{H}_{10} \\
\text { (Landolt-Bö rnstein (1979)) }\end{array}$ \\
\hline$\hat{\mathbf{C}}_{11}^{V, \text { Mon }}$ & 161 & 5.95 \\
\hline$\hat{\mathbf{C}}_{12}^{V, \text { Mon }}$ & 82 & 4.05 \\
\hline$\hat{\mathbf{c}}_{13}^{V, M o n}$ & 103 & 2.88 \\
\hline$\hat{\mathbf{C}}_{15}^{V, \text { Mon }}$ & -36 & 0.40 \\
\hline$\hat{\mathbf{c}}_{22}^{V, \text { Mon }}$ & 230 & 6.97 \\
\hline
\end{tabular}




\begin{tabular}{|l|l|l|}
\hline$\hat{\mathbf{c}}_{23}^{V, \text { Mon }}$ & 36 & 6.11 \\
\hline$\hat{\mathbf{c}}_{25}^{V, \text { Mon }}$ & 3 & 0.94 \\
\hline$\hat{\mathbf{c}}_{33}^{V, \text { Mon }}$ & 232 & 14.6 \\
\hline$\hat{\mathbf{c}}_{35}^{V, \text { Mon }}$ & -39 & 2.02 \\
\hline$\hat{\mathbf{c}}_{44}^{V, \text { Mon }}$ & 67.8 & 1.83 \\
\hline$\hat{\mathbf{c}}_{46}^{V, \text { Mon }}$ & 10 & -0.89 \\
\hline$\hat{\mathbf{c}}_{55}^{V, \text { Mon }}$ & 73.3 & 2.26 \\
\hline$\hat{\mathbf{c}}_{66}^{V, \text { Mon }}$ & 58.8 & 4.11 \\
\hline
\end{tabular}

Table 5.26:Reuss elastic constant data of monoclinic media

\begin{tabular}{|l|l|l|}
\hline Monoclinic Media & $\begin{array}{l}\text { Coesite,SiO } \\
\text { (Weidner and Carleton (1977)) }\end{array}$ & $\begin{array}{l}\text { Diphenyl, } \\
\mathbf{C}_{12} \mathbf{H}_{10} \\
\text { Landolt-Bö rnstein (1979)) }\end{array}$ \\
\hline$\hat{\mathbf{s}}_{11}^{R, \text { Mon }}$ & 11.3 & 283 \\
\hline$\hat{\mathbf{s}}_{12}^{R, \text { Mon }}$ & -3.50 & -184 \\
\hline$\hat{\mathbf{s}}_{13}^{R, \text { Mon }}$ & -3.90 & 19 \\
\hline$\hat{\mathbf{s}}_{15}^{R, \text { Mon }}$ & 3.60 & 9 \\
\hline$\hat{\mathbf{s}}_{22}^{R, \text { Mon }}$ & 5.30 & 346 \\
\hline$\hat{\mathbf{s}}_{23}^{R, \text { Mon }}$ & 0.40 & -107 \\
\hline$\hat{\mathbf{s}}_{25}^{R, \text { Mon }}$ & -1.70 & -16 \\
\hline$\hat{\mathbf{s}}_{33}^{R, \text { Mon }}$ & 6.20 & 118 \\
\hline$\hat{\mathbf{s}}_{35}^{R, \text { Mon }}$ & 1.40 & -65 \\
\hline$\hat{\mathbf{s}}_{44}^{R, \text { Mon }}$ & 611 \\
\hline$\hat{\mathbf{s}}_{46}^{R, \text { Mon }}$ & 15.1 & 132 \\
\hline$\hat{\mathbf{s}}_{55}^{R, \text { Mon }}$ & -2.60 & 509 \\
\hline$\hat{\mathbf{s}}_{66}^{R, \text { Mon }}$ & 16.2 & 272 \\
\hline
\end{tabular}

Table 5.27:Lower and upper bounds on effective eigenvalues of monoclinic media

\begin{tabular}{|l|l|l|}
\hline Monoclinic Media & Coesite,SiO & Diphenyl, $\mathbf{C}_{12} \mathbf{H}_{10}$ \\
\hline$\lambda_{1}^{R, \text { Mon }}$ & 2.76 & 0.051 \\
\hline$\lambda_{2}^{R, \text { Mon }}$ & 4.96 & 0.163 \\
\hline$\lambda_{3}^{R, \text { Mon }}$ & 12.5 & 0.521 \\
\hline$\lambda_{4}^{R, \text { Mon }}$ & 13.5 & 0.515 \\
\hline$\lambda_{5}^{R, \text { Mon }}$ & 19 & 0.656 \\
\hline$\lambda_{6}^{R, \text { Mon }}$ & 19.1 & 0.226 \\
\hline$\lambda_{1}^{V, \text { Mon }}$ & 52.3 & 1.52 \\
\hline$\lambda_{2}^{V, \text { Mon }}$ & 52.8 & 1.92 \\
\hline
\end{tabular}




\begin{tabular}{|l|l|l|}
\hline$\lambda_{3}^{V, \text { Mon }}$ & 74.3 & 1.94 \\
\hline$\lambda_{4}^{V, \text { Mon }}$ & 80 & 4.42 \\
\hline$\lambda_{5}^{V, \text { Mon }}$ & 201.5 & 6.13 \\
\hline$\lambda_{6}^{V, \text { Mon }}$ & 362.2 & 19.8 \\
\hline
\end{tabular}

\subsection{For Triclinic Media}

Table 5.28: Voigt elastic constant data of triclinic media

\begin{tabular}{|c|c|c|}
\hline Triclinic Media & $\begin{array}{l}\text { Ammonium tetroxalate } \\
\text { dihydrate(Küppers and Siegert (1970)) }\end{array}$ & $\begin{array}{l}\text { Potassium tetroxalate dihydrate } \\
\text { (Kü ppers and Siegert (1970)) }\end{array}$ \\
\hline$\hat{c}_{11}^{V, \text { Tric }}$ & 21.9 & 25.4 \\
\hline$\hat{c}_{12}^{V, \text { Tric }}$ & 12 & 11.8 \\
\hline$\hat{c}_{13}^{V, \text { Tric }}$ & 10.4 & 9.83 \\
\hline$\hat{c}_{14}^{V, \text { Tric }}$ & 1.60 & 0.72 \\
\hline$\hat{c}_{15}^{V, \text { Tric }}$ & 6 & 6.12 \\
\hline$\hat{c}_{16}^{V, \text { Tric }}$ & -1 & -1.23 \\
\hline$\hat{c}_{22}^{V, \text { Tric }}$ & 45.9 & 47.8 \\
\hline$\hat{c}_{23}^{V, \text { Tric }}$ & 16.3 & 14 \\
\hline$\hat{c}_{24}^{V, \text { Tric }}$ & 11.6 & 11.3 \\
\hline$\hat{c}_{25}^{V, \text { Tric }}$ & 2 & 1.46 \\
\hline$\hat{c}_{26}^{V, \text { Tric }}$ & -3.80 & -2.70 \\
\hline$\hat{c}_{33}^{V, \text { Tric }}$ & 36.4 & 34.3 \\
\hline$\hat{c}_{34}^{V, \text { Tric }}$ & 3.8 & 2.19 \\
\hline$\hat{c}_{35}^{V, \text { Tric }}$ & 2 & 1.47 \\
\hline$\hat{c}_{36}^{V, \text { Tric }}$ & -0.8 & 0.40 \\
\hline$\hat{c}_{44}^{V, \text { Tric }}$ & 10.4 & 10.2 \\
\hline$\hat{C}_{45}^{V, \text { Tric }}$ & 0.10 & -0.82 \\
\hline$\hat{c}_{46}^{V, \text { Tric }}$ & 0.10 & 0.53 \\
\hline$\hat{c}_{55}^{V, \text { Tric }}$ & 5.40 & 5.69 \\
\hline$\hat{c}_{56}^{V, \text { Tric }}$ & 0.10 & 0.70 \\
\hline$\hat{C}_{66}^{V, \text { Tric }}$ & 4.44 & 4.99 \\
\hline
\end{tabular}

Table 5.29:Reuss elastic constant data of triclinic media

\begin{tabular}{|l|l|l|}
\hline Triclinic Media & $\begin{array}{l}\text { Ammonium tetroxalate } \\
\text { dihydrate(Küppers and Siegert (1970)) }\end{array}$ & $\begin{array}{l}\text { Potassium tetroxalate dihydrate } \\
\text { Kü ppers and Siegert (1970)) }\end{array}$ \\
\hline$\hat{S}_{11}^{R, \text { Tric }}$ & 81.9 & 66.2 \\
\hline$\hat{S}_{12}^{R, \text { Tric }}$ & -15.2 & -10.2 \\
\hline$\hat{S}_{13}^{R, \text { Tric }}$ & -13 & -12.4 \\
\hline$\hat{S}_{14}^{R, \text { Tric }}$ & 9.80 & 2.8 \\
\hline
\end{tabular}




\begin{tabular}{|l|l|l|}
\hline$\hat{S}_{15}^{R, \text { Tric }}$ & -80.7 & -67.6 \\
\hline$\hat{S}_{16}^{R, \text { Tric }}$ & 5.80 & 20.9 \\
\hline$\hat{S}_{22}^{R, \text { Tric }}$ & 42 & 37.4 \\
\hline$\hat{S}_{23}^{R, \text { Tric }}$ & -9.80 & -9.80 \\
\hline$\hat{S}_{24}^{R, \text { Tric }}$ & -41.1 & -40.4 \\
\hline$\hat{S}_{25}^{R, \text { Tric }}$ & 5.30 & -4.80 \\
\hline$\hat{S}_{26}^{R, \text { Tric }}$ & 31.7 & 23.5 \\
\hline$\hat{S}_{33}^{R, \text { Tric }}$ & 35.2 & 36.1 \\
\hline$\hat{S}_{34}^{R, \text { Tric }}$ & 0.20 & 5.50 \\
\hline$\hat{S}_{35}^{R, \text { Tric }}$ & 4.90 & 8.90 \\
\hline$\hat{S}_{36}^{R, \text { Tric }}$ & -5.40 & -13.1 \\
\hline$\hat{S}_{44}^{R, \text { Tric }}$ & 140 & 146 \\
\hline$\hat{S}_{45}^{R, \text { Tric }}$ & 1.60 & 32.2 \\
\hline$\hat{S}_{46}^{R, \text { Tric }}$ & -37.5 & -41.6 \\
\hline$\hat{S}_{55}^{R, \text { Tric }}$ & 271 & 259 \\
\hline$\hat{S}_{56}^{R, \text { Tric }}$ & -20.9 & -59.5 \\
\hline$\hat{S}_{66}^{R, \text { Tric }}$ & 254 & 232 \\
\hline
\end{tabular}

Table 5.30:Lower and upper bounds on effective eigenvalues of triclinic media \begin{tabular}{|l|l|l}
\hline Triclinic Media Ammonium tetroxalate dihydrate & Potassium tetroxalate dihydrate
\end{tabular}

\begin{tabular}{|l|l|l|}
\hline$\lambda_{1}^{R, \text { Tric }}$ & 0.309 & 0.340 \\
\hline$\lambda_{2}^{R, \text { Tric }}$ & 0.266 & 0.198 \\
\hline$\lambda_{3}^{R, \text { Tric }}$ & 0.140 & 0.139 \\
\hline$\lambda_{4}^{R, \text { Tric }}$ & 0.015 & 0.015 \\
\hline$\lambda_{5}^{R, \text { Tric }}$ & 0.056 & 0.049 \\
\hline$\lambda_{6}^{R, \text { Tric }}$ & 0.039 & 0.036 \\
\hline$\lambda_{1}^{V, \text { Tric }}$ & 3.23 & 2.95 \\
\hline$\lambda_{2}^{V, \text { Tric }}$ & 3.76 & 5.05 \\
\hline$\lambda_{3}^{V, \text { Tric }}$ & 7.16 & 7.22 \\
\hline$\lambda_{4}^{V, \text { Tric }}$ & 17.8 & 20.4 \\
\hline$\lambda_{5}^{V, \text { Tric }}$ & 25.6 & 27.7 \\
\hline$\lambda_{6}^{V, \text { Tric }}$ & 67 & 65 \\
\hline
\end{tabular}

\section{Results}

The computed results in section 5 show that the materials selected randomly from same anisotropic elastic symmetry, depending upon the size of intervals between Reuss and Voigt bounds, can exhibit whether close to isotropy or anisotropy. In other words, for 
any anisotropic elastic symmetry, the larger interval between upper and lower bounds, states more anisotropy whereas smaller interval states more isotropic behavior. For some symmetry, the results are summarized as;

For cubic symmetry:

From tables 5.3 and 5.4, it is easy to see that Thallium manganase chloride is close to isotropy more than the other cubic materials. Since the intervals between Voigt and Reuss bounds on elastic constants of the material are very closer, the effective eigenvalues in terms of elastic constants are selected from a narrow range. On the other hand, Diamond exhibits the most anisotropy among the other cubic materials.

For tetragonal symmetry:

If tables 5.11 and 5.12 are examined, it is seen that Indium-cadmium alloy composed of \% 3.42 Cadmium and \% 96.58 Indium, is close to isotropy more than the others. As a result, the interval between bounds on Reuss and Voigt eigenvalues for the alloy is very small. Whereas Zircon shows the most anisotropy among the other materials.

For transversely isotropic symmetry:

According to calculated values in tables 5.15 and 5.16, Hafnium, Bone (dried phalanx) and Polystyrene are close to isotropy more than the other transversely isotropic materials. Because Voigt and Reuss bounds are very closer.

On the other hand, Zinc and Cobalt show the most anisotropy. Since the eigenvalues of Voigt and Reuss for Zinc and Cobalt are considerably different, their mechanical and elastic behaviors are expected to be more anisotropic. As a result, the effective eigenvalues in terms of elastic constants of them are selected from a large range.

For orthorhombic symmetry:

In tables 5.23 and 5.24, it is obvious to see that Maple and Human tibia are close to isotropy than the others. Because Voigt and Reuss bounds are very close to each other. So effective eigenvalues of these materials can be selected from a narrow range. Olivinite exhibits the most anisotropy among the other materials because of the large intervals between the corresponding bounds.

For monoclinic symmetry:

The intervals between Reuss and Voigt bounds on effective eigenvalues (given in table 5.27) for Diphenyl are closer than Coesite. The intervals between the corresponding Reuss and Voigt eigenvalues for Coesite are larger than Diphenyl. As a result, it can be said that Diphenyl is close to isotropy more than Coesite. The computed results are consistent with those calculated in the work of Dinçkal and Akgöz (2010).

\section{Conclusions}

In this paper, it has been shown that it is possible to construct bounds on the effective eigenvalues of any anisotropic elastic constants symmetry in terms of triclinic symmetry elastic by developing generalized Hill inequalities. Specific bounds have been presented for cubic, isotropic, transversely isotropic, tetragonal, trigonal, orthorhombic, monoclinic and triclinic symmetries. Constructing bounds on effective eigenvalues provides a deeper understanding about mechanical behavior of anisotropic materials. It also has significant effects on many applications in different fields such as: design of composite materials, examining the material symmetry types in detail, determination of materials which are highly anisotropic or close to isotropic, understanding the mechanical and elastic behaviour of natural composites such as bone and wood types. For instance wood as a composite and also an important structural engineering material has two types; hardwoods and softwoods, by computing eigenvalues and constructing bounds, it is seen that, softwoods are more anisotropic than hardwoods in other words, design of a wood-based product is critic due to this anisotropy.

\section{References}

Aleksandrov, K.S., Anistratov, A.T., Krupnyi, et al., 1975. X-Ray optical and ultrasound studies of phase-transitions in TLMNCL3. Fizika Tverdogo Tela, Vol.17, pp.735-740.

Akgö z, Y.C., Isc, C., Saunders, G.A., 1976. The elastic constants of antimony-25.5 at. \% arsenic alloy single crystals. Journal of Materials Science, Vol. 11, pp.291-296.

Bonfield, W., Grynpas, M.D., 1977. Anisotropy of Young's modulus of Bone, Nature (London), Vol. 270, pp.453-454.

Cowin, S.C., 1989. The mechanical properties of cortical bone. In: S.C. Cowin (ed.), Bone Mechanics. CRC Press, Boca Raton, FL.

Cowin, S.C. and Sadegh, A.M.,1991. Non-interacting modes for stress, strain and energy in anisotropic hard tissue, Journal of

Biomechanics, Vol.24, pp.859-867.

Dinçkal Ç. and Akgöz Y.C., 2010. Decomposition of elastic constant tensor into orthogonal parts, International Journal of

Engineering Science and Technology, Vol.2, No.6, pp.22-46.

Epstein, S. and De Bretteville, A.P.,1965. Elastic constants and wave propagation in Antimony and Bismuth, Physical Review, Vol.138, pp.A771.

Fisher, E.S., 1975. A review of solute effects on the elastic modulus of bec transition in Physics of Solid Solution Strengthening.

Proc. Symposium 1973 eds. E.W. Collings and H.L. Gegel., New York, Plenum Press, pp.195.

Fisher, E.S., Renken, C.J., 1964. Single-crystal elastic moduli + HCP to BCC transformation in TI, ZR, +HF. Physical Review A-

General Physics, Vol.135, pp.482. 
Gault, C., Boch, P. and A. Dauger, 1977. Variations of elastic-constants of Aluminium-Magnesium single-crystals with GuinierPreston Zones. Physica Status Solidi A-Applied Research, Vol.43, pp.625-632.

Graham, L.J.and R. Chang, 1975. Temperature and pressure-dependence of elastic properties of RBAG4I5. Journal of Applied Physics, Vol.46, pp.2433-2438.

Grimsditch, M.H. and A.K.Ramdas, 1975. Brillouin-scattering in Diamond. Physical Review B;Condensed Matter and Materials Physics, Vol.11, pp.3139-3148.

Haussü hl, S., 1965. Elastische und thermoelastiche Konstanten von $\mathrm{K}_{2} \mathrm{SO}_{4}, \mathrm{RB}_{2} \mathrm{SO}_{4}, \mathrm{CS}_{2} \mathrm{SO}_{4},\left(\mathrm{NH}_{4}\right)_{2} \mathrm{SO}_{4}$, $\mathrm{TI}_{2} \mathrm{SO}_{4}$ und $\mathrm{K}_{2} \mathrm{MG}_{2}\left(\mathrm{SO}_{4}\right)_{3}$. Acta Crystallographica, Vol.18, Part 5, pp.839.

Haussü hl, S., 1964. Elastische und thermoelastische eigenschaften von $\mathrm{KH}_{2} \mathrm{PO}_{4} \mathrm{KH}_{2} \mathrm{ASO}_{4} \mathrm{NH}_{4} \mathrm{H}_{2} \mathrm{PO}_{4} \mathrm{NH}_{4} \mathrm{H}$ $\mathrm{ASO}_{4}$ und $\mathrm{RBH}_{2} \mathrm{PO}_{4}$. Zeitschrift fur Kristallographie.Vol.120, pp.401.

Hill, R., 1963. Elastic properties of reinforced solids:some theoretical principles. Journal of the Mechanics and Physics of Solids, Vol.11, pp.357-372.

Humbert, P. and Plique, F., 1972. Elastic properties of monocrystalline rhombohedral carbonates-Calcite, Magnesite, Dolomite. Comptes Rendus Hebdomadaines des Sé ances de l Academie des Sciences Serie B, Vol.275, pp.391.

Kollmann, F.F.P. and Cô té , 1968. Principal of Wood Science and Technology, I. Solid Wood. Springer, Berlin.

Küppers, H. and Siegert, H.,1970. Elastic constants of triclinic crystals Ammonium and Potassium Tetroxalate Dihydrate. Acta Crystallographica Section A, Vol.26, pp.401.

Landolt-Bö rnstein, 1979.Numerical Data and Functional Relationships in Science and Technology, New Series, Group III, (Crystal and Solid State Physics), Vol.11, Springer-Verlag, Berlin.

Macfarlane, R.E., Rayne, J.A., and C.K. Jones, 1965. Anomalous temperature dependence of shear modulus C44 for Platinum, Physics Letters, Vol.18, pp.91.

Madhava, M.R., and Saunders, G.A., 1977.Ultrasonic study of elastic phase-transition in In-Cd alloys, Philosophical Magazine,Vol.36, pp.777-796.

Martin, R.M., 1972. Relation between elastic tensors of Wurtzite and Zincblende structure materials. Physical Review, Vol. B6, pp.4546-4553.

Masumoto, H., Saito, H. and Kikuchi, M., 1967. Thermal expansion and temperature dependence of Young's Modulus of single crystals of hexagonal, Cobalt. Science Reports of the Research Institutes Tohoku University A- Physics Chemistry and Metallurgy, Vol.19, pp.172.

Mehrabadi, M.M., Cowin, S.C., 1995. Anisotropic symmetries of linear elasticity. Appl. Mech. Rev., Vol.48, pp.247-285.

Pace, N.G. and Saunders G.A.,1971. Elastic wave propagation in group-VB semimetals. Journal of Physics and Chemistry of Solids, Vol. 32, pp.1585.

Robrock, K.H. and W.Schilling, 1976. Diaelastic modulus change of aluminium after low-temperature electron-irradiation. Journal of Physics F-Metal Physics, Vol.6, pp.303-314.

Shimizu, H., Tsukamoto, M., Ishibashi, Y. and Umeno, M.,1974. Brillouin-scattering in Sodium Nitrite. Journal of the Physical Society of Japan, Vol.36, pp.498-503.

Singh, D.P., Singh, S. and Chendra S., 1977. Indian J. Phys., Vol.A 51, pp.97.

Strang, G.,1976. Linear Algebra and Its Applications, 3rd edition, Academic Press.

Ting, T.C.T., 1996.Positive definiteness of anisotropic elastic constants. Mathematics and Mechanics of Solids, Vol.1, pp.301-314.

Van Der Planken, J., Greiner, J.D. and Smith, J.F.,1971. Magnetic susceptibilities and single crystalline elastic constants of LeadIndium alloys. Journal of Materials Science., Vol.6, pp.1331.

Weidner, D.J. and Carleton, H.R., 1977. Elasticity of Coesite. Journal of Geophysical Research, Vol.82, pp.1334-1346.

Wright, A., Faraday, C.S.N., White, E.F.T., et al., 1971. Elastic constants of oriented glassy polymers. Journal of Physics DApplied Physics, Vol.4, pp.2002.

\section{Biographical notes}

Dr. Ç. Dinçkal is Assistant Professor at the Department of Civil Engineering in Çankaya University, Turkey. She also gives lectures in engineering faculty of Ankara University. She graduated from department of industrial engineering, Çankaya University, Ankara, Turkey. She received her M.Sc. and Ph.D degree from Department of Engineering Sciences, Middle East Technical University, Ankara, Turkey. Her areas of interest include tensors, anisotropy of crystals, mechanics of composite materials and computational mechanics.

Received April 2011

Accepted June 2011

Final acceptance in revised form June 2011 